\title{
HIGH-ORDER WELL-BALANCED FINITE-VOLUME SCHEMES FOR HYDRODYNAMIC EQUATIONS WITH NONLOCAL FREE ENERGY*
}

\author{
JOSÉ A. CARRILLO ${ }^{\dagger}$, MANUEL J. CASTRO $^{\ddagger}$, SERAFIM KALLIADASIS $^{\S}$, AND \\ SERGIO P. PEREZ
}

\begin{abstract}
We propose high-order well-balanced finite-volume schemes for a broad class of hydrodynamic systems with attractive-repulsive interaction forces and linear and nonlinear damping. Our schemes are suitable for free energies containing convolutions of an interaction potential with the density, which are essential for applications such as the Keller-Segel model, more general Euler-Poisson systems, or dynamic-density functional theory. Our schemes are also equipped with a nonnegative-density reconstruction which allows for vacuum regions during the simulation. We provide several prototypical examples from relevant applications highlighting the benefit of our algorithms and also elucidate some of our analytical results
\end{abstract}

Key words. well-balanced scheme, hydrodynamic system, nonlocal free energy, high-order scheme, finite-volume scheme

AMS subject classifications. 65XX, 35Qxx, 35Q35, 35Q82, 76M12

DOI. $10.1137 / 20 \mathrm{M} 1332645$

1. Introduction. Well-balanced schemes have emerged as a paramount tool to simulate systems governed by balance/conservation laws. This is due to their ability to numerically preserve steady states and resolve small perturbations of those states even with coarse meshes. Well-balanced schemes were introduced nearly three decades ago with the initial works by Bermúdez and Vázquez [5], Greenberg and Leroux [52] and Gosse [49]. One of the most popular applications for well-balanced schemes from the very beginning has been the shallow-water equations. Further contributions of note are the hydrostatic reconstruction in $[1,25]$ together with application scenarios where well-balanced schemes have proven quite successful: tsunami propagation [27], coastal hydrodynamics [63], and irregular topographies [42], to name but a few. Inspired by the strong results for the shallow-water equations, plenty of authors have successfully employed well-balanced schemes in a plethora of balance-law problems from wave

*Submitted to the journal's Methods and Algorithms for Scientific Computing section April 20, 2020; accepted for publication (in revised form) November 18, 2020; published electronically March $4,2021$.

https://doi.org/10.1137/20M1332645

Funding: José A. Carrillo was partially supported by EPSRC grant EP/P031587/1 and the Advanced Grant Nonlocal-CPD (Nonlocal PDEs for Complex Particle Dynamics: Phase Transitions, Patterns and Synchronization) of the European Research Council Executive Agency (ERC) under the European Union's Horizon 2020 research and innovation programme through grant agreement 883363. Manuel J. Castro acknowledges financial support from the Spanish Government and FEDER through the research project RTI2018-096064-B-C2(1/2), the Junta de Andalucía research projects P18-RT-3163 and UMA18-FEDERJA-161. Serafim Kalliadasis acknowledges financial support from the EPSRC through grant EP/L020564. Sergio P. Perez acknowledges financial support from the Imperial College President's PhD Scholarship.

${ }^{\dagger}$ Mathematical Institute, University of Oxford, Oxford, OX2 6GG United Kingdom (carrillo@ maths.ox.ac.uk).

${ }^{\ddagger}$ Departamento Análisis Matemático, Estadística e Investigación Operativa y Matemática Aplicada, Universidad de Málaga, Bulevar Louis Pasteur, 31, 29010 Málaga, Spain (mjcastro@uma.es).

$\S$ Department of Chemical Engineering, Imperial College London, London SW7 2AZ, United Kingdom (s.kalliadasis@imperial.ac.uk).

\Corresponding author. Departments of Chemical Engineering and Mathematics, Imperial College London, London, SW7 2AZ United Kingdom (sergio.perez15@imperial.ac.uk).

$$
\text { A828 }
$$


propagation in elastic media [79] and chemosensitive movement of cells [40] to flow through a nozzle [44] and the Euler equations with gravity [59, 78]. Recently, a general procedure to construct high-order well-balanced schemes for one-dimensional balance laws was described in [26].

In our previous work [20] we extended the applicability of well-balanced schemes to the broad class of hydrodynamic models with attractive-repulsive interaction forces. In particular, we considered interactions associated with nonlocal convolutions or functions of convolutions, which is commonplace in applications such as the Keller-Segel model [9], more general Euler-Poisson systems [55], or in dynamic-density functional theory (DDFT) [47, 48]. This class of balance laws may contain linear or nonlinear damping effects, such as the Cucker-Smale alignment term in collective behavior [36]. The corresponding hydrodynamic systems have the general form

$$
\left\{\begin{array}{l}
\partial_{t} \rho+\nabla \cdot(\rho \boldsymbol{u})=0, \quad \boldsymbol{x} \in \mathbb{R}^{d}, \quad t>0 \\
\partial_{t}(\rho \boldsymbol{u})+\nabla \cdot(\rho \boldsymbol{u} \otimes \boldsymbol{u})=-\rho \nabla \frac{\delta \mathcal{F}[\rho]}{\delta \rho}-\gamma \rho \boldsymbol{u}-\rho \int_{\mathbb{R}^{d}} \psi(\boldsymbol{x}-\boldsymbol{y})(\boldsymbol{u}(\boldsymbol{x})-\boldsymbol{u}(\boldsymbol{y})) \rho(\boldsymbol{y}) d \boldsymbol{y}
\end{array}\right.
$$

where the free-energy functional $\mathcal{F}[\rho]$ contains the pressure $P(\rho)$ and generic potential terms $H(\boldsymbol{x}, \rho)$ and can be decomposed as

$$
-\rho \nabla \frac{\delta \mathcal{F}}{\delta \rho}=-\nabla P(\rho)-\rho \nabla H(\boldsymbol{x}, \rho) .
$$

The potential terms $H(\boldsymbol{x}, \rho)$ involve an external field $V(x)$ and an interaction potential $W(x)$ convoluted with the density $\rho$, so that

$$
H(\boldsymbol{x}, \rho)=V(\boldsymbol{x})+W(\boldsymbol{x}) \star \rho .
$$

Finally, the free-energy functional has the form

$$
\mathcal{F}[\rho]=\int_{\mathbb{R}^{d}} \Pi(\rho) d \boldsymbol{x}+\int_{\mathbb{R}^{d}} V(\boldsymbol{x}) \rho(\boldsymbol{x}) d \boldsymbol{x}+\frac{1}{2} \int_{\mathbb{R}^{d}} \int_{\mathbb{R}^{d}} W(\boldsymbol{x}-\boldsymbol{y}) \rho(\boldsymbol{x}) \rho(\boldsymbol{y}) d \boldsymbol{x} d \boldsymbol{y},
$$

where $\rho \Pi^{\prime \prime}(\rho)=P^{\prime}(\rho)$. The steady states of the system (1.1), whose preservation at the discrete level is the main aim of the design of well-balanced schemes, are characterized by

$(1.3)$

$\frac{\delta \mathcal{F}}{\delta \rho}=\Pi^{\prime}(\rho)+H(\boldsymbol{x}, \rho)=$ constant on each connected component of $\operatorname{supp}(\rho)$ and $u=0$,

where the constant can vary on different connected components of $\operatorname{supp}(\rho)$. These steady states are stationary $(u=0)$ due to the dissipation of the linear damping $-\gamma \rho u$ or nonlinear damping in the system (1.1), which ensures that the momentum eventually vanishes. This is due to the fact that the total energy of the system, defined as the sum of kinetic energy and free energy,

$$
E(\rho, \boldsymbol{u})=\int_{\mathbb{R}^{d}} \frac{1}{2} \rho|\boldsymbol{u}|^{2} d \boldsymbol{x}+\mathcal{F}(\rho)
$$

is formally dissipated (see $[15,21,46])$ as

$$
\frac{d E(\rho, \boldsymbol{u})}{d t}=-\gamma \int_{\mathbb{R}^{d}} \rho|\boldsymbol{u}|^{2} d \boldsymbol{x}-\int_{\mathbb{R}^{d}} \int_{\mathbb{R}^{d}} \psi(\boldsymbol{x}-\boldsymbol{y})|\boldsymbol{u}(\boldsymbol{y})-\boldsymbol{u}(\boldsymbol{x})|^{2} \rho(\boldsymbol{x}) \rho(\boldsymbol{y}) d \boldsymbol{x} d \boldsymbol{y} .
$$


Furthermore, the system (1.1) also satisfies an entropy identity

$$
\begin{aligned}
\partial_{t} \eta(\rho, \rho \mathbf{u})+\nabla \cdot \boldsymbol{G}(\rho, \rho \mathbf{u})= & -\rho \boldsymbol{u} \cdot \nabla H(\boldsymbol{x}, \rho) \\
& -\gamma \rho|\boldsymbol{u}|^{2}-\rho \int_{\mathbb{R}^{d}} \psi(\boldsymbol{x}-\boldsymbol{y}) \boldsymbol{u}(\boldsymbol{x}) \cdot(\boldsymbol{u}(\boldsymbol{x})-\boldsymbol{u}(\boldsymbol{y})) \rho(\boldsymbol{y}) d \boldsymbol{y},
\end{aligned}
$$

where $\eta(\rho, \rho \boldsymbol{u})$ and $\boldsymbol{G}(\rho, \rho \boldsymbol{u})$ are the entropy and the entropy flux defined as

$$
\eta(\rho, \rho \boldsymbol{u})=\rho \frac{|\boldsymbol{u}|^{2}}{2}+\Pi(\rho), \quad \boldsymbol{G}(\rho, \rho \boldsymbol{u})=\rho \boldsymbol{u}\left(\frac{|\boldsymbol{u}|^{2}}{2}+\Pi^{\prime}(\rho)\right) .
$$

In addition to the well-balanced property, many authors have sought to construct numerical schemes that preserve the structural properties of the system (1.1) during its temporal evolution. These endeavors have aimed to satisfy discretely the entropy identity (1.6) as well as the dissipation relations for the total energy in (1.5), both for the original system (1.1) and its overdamped versions. We refer the reader to $[2,41,77]$ for more information about entropy stable schemes and to $[3,12]$ for useful insights into energy dissipating schemes. The well-balanced finite-volume scheme of our previous work [20] was designed to be at the same time well-balanced, entropy stable, and energy dissipating, though only for first- and second-order accuracy.

The main contribution of the present work is to extend our previous scheme for the system (1.1) from first and second order to high order. Several authors have already proposed high-order well-balanced schemes for systems where the potential terms in the free energy (1.2) are local, such as the shallow-water equations [23, 24, 28, 31, 68], chemotaxis [40], and other applications [79]. These schemes rely on finite differences, finite volumes, or discontinuous Galerkin approaches, and, specially for the shallow-water case, there have been plenty of contributions devoted to particular configurations and scenarios: presence of dry areas and bottom topography [42], tsunami propagation [27], traffic flow model [28], moving steady states [24, 30, 69], etc. In future work we aim to explore the straightforward extension of our well-balanced scheme to multidimensional problems.

Here we consider the much broader class of free energies in (1.2), which include interaction potentials leading to forces given by the convolution with the density $\rho$ and possible linear or nonlinear damping effects from the field of collective behavior. Applications of this type include the Keller-Segel model, generalized Euler-Poisson systems [55], and DDFT [47, 48]. We complement our high-order finite-volume schemes with the desired properties of well-balancing and the nonnegativity of the density, which allows for vacuum regions in the simulations. It is worth mentioning that previous works have already addressed the construction of high-order well-balanced schemes for diverse types of steady state relations. Nevertheless the technical challenge here is to consider nonlinear and nonlocal steady states that involve convolutions. The well-balanced methodology for shallow-water equations, for instance, is not directly applicable to our steady states.

Our work lays the foundations for the construction of well-balanced high-order schemes that may satisfy further fundamental properties of the system (1.1), such as the discrete versions of the energy dissipation in (1.5) and entropy identity in (1.6), or even the well-balanced property for the challenging moving steady states. Developing schemes, amongst the class of positivity-preserving high-order schemes introduced in the present work, satisfying also the entropy stability and energy dissipating proper- 
ties, is a challenging open question. However, in section 3 we show that the simulations satisfy the discrete energy dissipation in practice.

The paper is structured as follows. First, in section 2 we explain the construction of our well-balanced high-order finite-volume scheme. In subsection 2.1 we begin by recalling our first-order numerical scheme from [20], and then in subsection 2.2 we provide a first-attempt to extend such a scheme to high order. The correct wellbalanced formulation for that high-order scheme is provided in subsection 2.3. The last subsection 2.4 summarizes algorithmic implementation of the scheme. Second, in section 3 we depict a battery of simulations for relevant applications of system (1.1). In subsection 3.1 we numerically check the well-balanced property and high-order accuracy of our scheme, and subsequently in subsection 3.2 we tackle applications for varied choices of the free energy, leading to interesting numerical experiments for which analytical results are limited in the literature.

2. High-order well-balanced finite-volume scheme. The different terms of the one-dimensional system (1.1) are usually gathered in the form of

$$
\partial_{t} U+\partial_{x} F(U)=S_{H}(U, H)+S_{D}(x, U)
$$

with

and

$$
U=\left(\begin{array}{c}
\rho \\
\rho u
\end{array}\right), \quad F(U)=\left(\begin{array}{c}
\rho u \\
\rho u^{2}+P(\rho)
\end{array}\right)
$$

$S_{H}(U, H)=\left(\begin{array}{c}0 \\ -\rho \partial_{x} H\end{array}\right), \quad S_{D}(x, U)=\left(\begin{array}{c}0 \\ -\gamma \rho u-\rho \int_{\mathbb{R}} \psi(x-y)(u(x)-u(y)) \rho(y) d y\end{array}\right)$,

where the $U$ are the unknown variables, $F(U)$ the fluxes, and $S_{H}(U, H)$ and $S_{D}(x, U)$ are the sources related to forces with potential $H$ and damping terms, respectively. In what follows, we only consider the source term $S_{H}(U, H)$ due to the forces as we focus on the definition of a well-balanced high-order scheme for stationary solutions (1.3). At the end of this section we propose a high-order discretization of the source damping term $S_{D}(x, U)$ that vanishes at stationary states.

We consider a mesh composed by cells $\left[x_{i-1 / 2}, x_{i+1 / 2}\right], 1 \leq i \leq N$, whose length $\Delta x$ is supposed to be constant for simplicity. Let us denote with $U_{i}(t)$ the approximation of the average of the exact solution at the $i$ th cell, $\left[x_{i-1 / 2}, x_{i+1 / 2}\right]$, at time $t$

$$
U_{i}(t)=\sum_{j=1}^{n_{s}} \alpha_{j} U\left(x_{i}^{j}, t\right) \cong \frac{1}{\Delta x} \int_{x_{i-1 / 2}}^{x_{i+1 / 2}} U(x, t) d x
$$

and we denote by $H_{i}(t)$ the approximation of the cell average of $H(x, \rho)=V(x)+$ $W(x) \star \rho$ at time $t$,

$$
H_{i}(t)=\sum_{j=1}^{n_{s}} \alpha_{j} H\left(x_{i}^{j}, \rho\left(x_{i}^{j}, t\right)\right) \cong \frac{1}{\Delta x} \int_{x_{i-1 / 2}}^{x_{i+1 / 2}} H(x, \rho(x, t)) d x .
$$

In the previous expressions we denote as $\alpha_{j}$ and $x_{i}^{j}$, for $j=1, \ldots, n_{s}$, the weights and quadrature points of a particular high-order quadrature formula for the cell $\left[x_{i-1 / 2}, x_{i+1 / 2}\right]$. In this work we employ the fifth-order standard Gaussian quadrature.

As pointed out in the introduction, one of the main contributions of this work is to construct high-order well-balanced schemes for free energies that may depend on the 
convolution of the density and an interaction potential, $W(x) \star \rho$. These convolutions are included in the steady state relations in (1.3), but for the discrete version of these relations one has to approximate the convolutions by a high-order quadrature formula. In the next definition we clarify the concept of well-balanced scheme for this kind of free energies.

Definition 2.1 (well-balanced scheme). We consider a semidiscrete method to approximate (2.1),

$$
\left\{\begin{aligned}
\frac{d U_{i}}{d t} & =-\frac{1}{\Delta x} \mathcal{L}\left(\Delta x, U_{j}(t), H_{j}(t), j \in \mathcal{S}_{i}\right), \\
U(0) & =U_{0}
\end{aligned}\right.
$$

where $U(t)=\left\{U_{i}(t)\right\}_{i=1}^{N}$ represents the vector of the approximations of the averaged values of the exact solutions at time $t, U_{0}=\left\{U_{i}(0)\right\}$ is the vector of the initial conditions, and $\mathcal{S}_{i}$ the stencil of the numerical scheme.

Now let us assume that $u(x)=0, \rho(x)$ is a smooth function, and $H_{\Delta x}(x)$ is a discrete approximation of $H=V+W \star \rho$ with the form

$$
H_{\Delta x}(x)=V(x)+\Delta x \sum_{l=1}^{M} \sum_{m=1}^{n_{s}} \alpha_{m} W\left(x-x_{l}^{m}\right) \rho\left(x_{l}^{m}\right)
$$

and satisfying

$$
\Pi^{\prime}(\rho(x))+H_{\Delta x}(x)=C_{\Gamma} \text { in each } \Lambda_{\Gamma}, \Gamma \in \mathbb{N},
$$

where $\Lambda_{\Gamma}, \Gamma \in \mathbb{N}$, denotes the possible infinite sequence indexed by $\Gamma$ of subsets $\Lambda_{\Gamma}$ of subsequent indices $i \in\{1, \ldots, N\}$, where $\rho(x)>0$ and $u=0$, and $C_{\Gamma}$ is the corresponding constant in that connected component of the discrete support.

It then follows that the semidiscrete numerical scheme (2.3) is said to be wellbalanced for

$$
U=\left(\begin{array}{c}
\rho(x) \\
0
\end{array}\right) \text { and } H_{\Delta x}
$$

if the vector of their approximated averages is a critical point of (2.3), i.e.,

$$
\mathcal{L}\left(\Delta x, U_{j}, H_{j}, j \in \mathcal{S}_{i}\right)=0,1 \leq i \leq N,
$$

where

$$
U_{i}=\sum_{j=1}^{n_{s}} \alpha_{j} U\left(x_{i}^{j}\right) \quad \text { and } \quad H_{i}=\sum_{j=1}^{n_{s}} \alpha_{j} H_{\Delta x}\left(x_{i}^{j}\right) .
$$

In what follows we begin by briefly recalling in subsection 2.1 the first-order well-balanced scheme for (2.1) introduced in [20], which serves as a starting point to construct high-order schemes by employing a high-order reconstruction operator, as described in subsection 2.2. Then in subsection 2.3 we describe how to adapt these high-order schemes so that they are well-balanced in the sense defined in 2.1.

2.1. First-order numerical scheme. The first-order semidiscrete well-balanced finite-volume scheme for system (2.1) introduced in [20] can be written as

$$
\frac{d U_{i}}{d t}=-\frac{1}{\Delta x}\left(\mathbb{F}_{i+1 / 2}^{-}-\mathbb{F}_{i-1 / 2}^{+}\right),
$$

where $\mathbb{F}_{i+1 / 2}^{ \pm}$is defined using a standard consistent numerical flux for the homogeneous system applied to the so-called hydrostatic-reconstructed states, with an extra term ensuring the consistency of the numerical scheme (2.5) applied to system (2.1), as 
well as its well-balanced character. In [20] the midpoint quadrature formula is used to approximate both the cell averages of the exact solution and $H_{\Delta x}(x)$. In what follows we suppress the time dependence on the cell averages for simplicity. We define $\mathbb{F}_{i+1 / 2}^{ \pm}$in terms of the cell averages $U_{i}, U_{i+1}, H_{i}$, and $H_{i+1}$ as

$$
\mathbb{F}_{i+1 / 2}^{ \pm}\left(U_{i}, U_{i+1}, H_{i}, H_{i+1}\right)=\mathcal{F}\left(U_{i+1 / 2}^{H R,-}, U_{i+1 / 2}^{H R,+}\right) \pm S_{i+1 / 2}^{H R, \pm},
$$

where $\mathcal{F}(U, V)$ is the standard local Lax-Friedrich numerical flux for the homogeneous system,

$$
\mathcal{F}(U, V)=\frac{1}{2}(F(U)+F(V)-|\lambda(U, V)|(V-U)),
$$

where $|\lambda(U, V)|$ is a bound for the maximum absolute value of the wave speeds for the Riemann problem with the constant states $U$ and $V$.

In [20] we employ the hydrostatic reconstruction first introduced in [1] in the context of shallow-water equations. Here we denote the hydrostatic-reconstructed states as $U_{i+1 / 2}^{H R, \pm}$, and we compute them as follows:

(1) First an intermediate state $H_{i+1 / 2}$ is computed as

$$
H_{i+1 / 2}=\max \left(H_{i+1}, H_{i}\right) .
$$

(2) Next, we define the hydrostatic-reconstructed states as

$$
U_{i+1 / 2}^{H R, \pm}=\left(\begin{array}{c}
\rho_{i+1 / 2}^{H R, \pm} \\
(\rho u)_{i+1 / 2}^{H R, \pm}
\end{array}\right)
$$

where

$$
\begin{gathered}
\rho_{i+1 / 2}^{H R,-}=\xi\left(\Pi^{\prime}\left(\rho_{i}\right)+H_{i}-H_{i+1 / 2}\right), \quad(\rho u)_{i+1 / 2}^{H R,-}=\rho_{i+1 / 2}^{H R,-} u_{i}, \\
\rho_{i+1 / 2}^{H R,+}=\xi\left(\Pi^{\prime}\left(\rho_{i+1}\right)+H_{i+1}-H_{i+1 / 2}\right), \quad(\rho u)_{i+1 / 2}^{H R,+}=\rho_{i+1 / 2}^{H R,+} u_{i+1},
\end{gathered}
$$

with $\xi(s)$ being the inverse function of $\Pi^{\prime}(s)$ for $s>0$ and $u_{i}=(\rho u)_{i} / \rho_{i}$.

The last ingredients for the flux in (2.6) are the terms $S_{i+1 / 2}^{H R, \pm}$, which correspond to the correction introduced in the numerical scheme to guarantee consistency and well-balanced properties (see $[1,20]$ ),

$$
S_{i+1 / 2}^{H R,+}=\left(\begin{array}{c}
0 \\
P\left(\rho_{i+1}\right)-P\left(\rho_{i+1 / 2}^{H R,+}\right)
\end{array}\right) \quad \text { and } \quad S_{i+1 / 2}^{H R,-}=\left(\begin{array}{c}
0 \\
P\left(\rho_{i+1 / 2}^{H R,-}\right)-P\left(\rho_{i}\right)
\end{array}\right) .
$$

It is straightforward to check that the semi-discrete numerical scheme (2.5)-(2.9) is well-balanced in the sense defined in definition 2.1 (see [20]). It may also be surprising that the potential cell averages $H_{i}$ do not appear in the source terms (2.9), but this is a consequence of the well-balanced methodology and we refer the reader to $[1,8,20]$ for further details.

2.2. High-order extension. The basic ingredients to design a high-order finitevolume method for system $(2.1)$, assuming $S_{D}(x, U)=0$, are

- a consistent first-order numerical flux for system (2.1), like the one proposed in [20] and described in the previous subsection; 
- a high-order reconstruction operator, i.e., an operator that, given a family of cell values $\left\{U_{i}(t)\right\}$, provides at every cell $\left[x_{i-1 / 2}, x_{i+1 / 2}\right]$ a smooth function that depends on the values at some neighbor cells whose indices belong to the so-called stencil $\mathcal{S}_{i}$ :

$$
R_{i}^{U}(x)=R_{i}^{U}\left(x ;\left\{U_{j}(t)\right\}_{j \in \mathcal{S}_{i}}\right),
$$

so that $R_{i}^{U}(x)$ is a high-order approximation of $U(x, t)$ in the $i$ th cell at time $t$. Here we use third- and fifth-order compact weighted essentially nonoscillatory (CWENO) reconstruction operators [11, 61, 62]. The main advantage of CWENO compared to weighted essentially nonoscillatory (WENO) reconstruction operators (see [74-76]) is that CWENO reconstructions achieve uniform high-order approximation in the entire cell, while WENO reconstruction operators are proposed to achieve high-order approximation at the boundaries of the cell. Thus, standard WENO-5 reconstructions achieves 5th-order accuracy at the boundaries of the cell, while it is only 3rd-order at the interior points. Therefore, CWENO reconstruction operators are specially useful in balance laws such as (2.1), where the source term has to be evaluated at inner points of the cell. We complement the CWENO reconstruction operators with the positive-density limiters from [82] to ensure physical admissible reconstructed values for the density. For further details we refer the reader to Appendix SM1.

Using these ingredients, one could consider a high-order finite-volume semidiscrete numerical method of the form

$$
\frac{d U_{i}}{d t}=-\frac{1}{\Delta x}\left(\mathbb{F}_{i+1 / 2}^{-}-\mathbb{F}_{i-1 / 2}^{+}\right)+\frac{1}{\Delta x} \int_{x_{i-1 / 2}}^{x_{i+1 / 2}} S_{H}\left(R_{i}^{U}(x), R_{i}^{H}(x)\right) d x,
$$

where

- $R_{i}^{U}(x)$ and $R_{i}^{H}(x)$ are the approximations of the solution $U(x, t)$ and the function $H_{\Delta x}(x)$, respectively, at the $i$ th cell given by some high-order reconstruction operators from the sequence of cell values $\left\{U_{i}(t)\right\}$ and $\left\{H_{i}(t)\right\}$, respectively, i.e.,

$$
R_{i}^{U}(x)=R_{i}\left(x ;\left\{U_{j}(t)\right\}_{j \in \mathcal{S}_{i}}\right) \quad \text { and } \quad R_{i}^{H}(x)=R_{i}\left(x ;\left\{H_{j}(t)\right\}_{j \in \mathcal{S}_{i}}\right) ;
$$

- $\mathbb{F}_{i+1 / 2}^{ \pm}$is the numerical flux defined in (2.6) applied to the reconstructed states $U_{i+1 / 2}^{\mp}$ and $H_{i+1 / 2}^{\mp}$, i.e.,

$$
\mathbb{F}_{i+1 / 2}^{ \pm}=\mathbb{F}\left(U_{i+1 / 2}^{-}, U_{i+1 / 2}^{+}, H_{i+1 / 2}^{-}, H_{i+1 / 2}^{+}\right)
$$

with

$$
U_{i+1 / 2}^{-}=R_{i}^{U}\left(x_{i+1 / 2}\right), \quad U_{i+1 / 2}^{+}=R_{i+1}^{U}\left(x_{i+1 / 2}\right),
$$

and

$$
H_{i+1 / 2}^{-}=R_{i}^{H}\left(x_{i+1 / 2}\right), \quad H_{i+1 / 2}^{+}=R_{i+1}^{H}\left(x_{i+1 / 2}\right) .
$$

Following the procedure described in [22, Theorem 3.2] and [23, section 4], it is possible to prove that the semidiscrete numerical scheme (2.10) is a high-order numerical scheme of order $p>1$, if the following three conditions are satisfied:

(i) $H_{\Delta x}(x)$ is a high-order approximation of $H(x, \rho)$ of order $p>1$;

(ii) $R_{i}^{U}(x)$ and $R_{i}^{H}(x)$ are high-order reconstruction operators of order at least $p>1$ 
(iii) the volume integral

$$
\frac{1}{\Delta x} \int_{x_{i-1 / 2}}^{x_{i+1 / 2}} S_{H}\left(R_{i}^{U}(x), R_{i}^{H}(x)\right) d x
$$

is computed exactly or approximated with a quadrature formula of order greater than or equal to $p>1$.

Unfortunately, when employing standard (CWENO, WENO, ...) reconstructions the resulting numerical scheme is, in general, not well-balanced. Indeed, if $\left\{U_{i}\right\}_{i=1}^{N}$ and $\left\{H_{i}\right\}_{i=1}^{N}$ are the cell averages of a discrete steady state satisfying (2.4), then their reconstructions do not necessary satisfy the discrete relations

$$
\Pi^{\prime}\left(R_{i}^{\rho}(x)\right)+R_{i}^{H}(x)=C, 1 \leq i \leq N .
$$

In the previous expression we assume, for simplicity, that we have only one connected component.

In what follows we aim to propose a modified reconstruction procedure which respects (2.11) for any discrete steady state satisfying (2.4). Thanks to this modification we can prove that our scheme is both high-order accurate and well-balanced.

2.3. High-order well-balanced numerical scheme. Let us assume that the sequences of cell averages $\left\{U_{i}\right\}_{i=1}^{N}$ and $\left\{K_{i}\right\}_{i=1}^{N}$ are known with

$$
U_{i}=\left(\begin{array}{c}
\rho_{i}=\sum_{j=1}^{n_{s}} \alpha_{j} \rho\left(x_{i}^{j}\right) \\
(\rho u)_{i}=\sum_{j=1}^{n_{s}} \alpha_{j}(\rho u)\left(x_{i}^{j}\right)
\end{array}\right)
$$

and

$$
K_{i}=\sum_{j=1}^{n_{s}} \alpha_{j}\left[\Pi^{\prime}\left(\rho\left(x_{i}^{j}\right)\right)+H_{\Delta x}\left(x_{i}^{j}\right)\right] .
$$

For such cell averages we propose the following reconstruction procedure:

- We consider a standard high-order reconstruction operator for the conserved variables $\rho$ and $\rho u$, and also applied to the sequence $\left\{K_{i}\right\}_{i=1}^{N}$,

$$
\begin{gathered}
R_{i}^{\rho}(x)=R_{i}\left(x,\left\{\rho_{j}\right\}_{j \in S_{i}}\right), \\
R_{i}^{\rho u}(x)=R_{i}\left(x,\left\{(\rho u)_{j}\right\}_{j \in S_{i}}\right), \\
R_{i}^{K}(x)=R_{i}\left(x,\{K\}_{j \in S_{i}}\right) ;
\end{gathered}
$$

- the reconstruction operator for $H_{\Delta x}(x)$ is defined as

$$
R_{i}^{H}(x)=R_{i}^{K}(x)-\Pi^{\prime}\left(R_{i}^{\rho}(x)\right)
$$

with $R_{i}^{H}$ not being a polynomial since it depends on the function $\Pi^{\prime}(\rho)$.

The previous reconstruction procedure satisfies the following property.

TheOREM 2.2. Let $u=0, \rho(x)$ and $H_{\Delta x}(x)$ be satisfying (2.4), that is $\rho, u=0$ is a discrete stationary solution of system (2.1), then the reconstructions $R_{i}^{\rho}(x), R_{i}^{\rho u}(x)$, and $R_{i}^{H}(x)$ are discrete stationary solutions of system $(2.1)$ at $\left[x_{i-1 / 2}, x_{i+1 / 2}\right]$. 
Proof. Let us suppose for simplicity that the stationary solution is only defined in one connected component. Therefore, $K_{i}=C, 1 \leq i \leq N$.

As standard reconstruction operators like CWENO are exact for constant functions, we have that $R_{i}^{\rho u}(x)=0$ and $R_{i}^{K}(x)=C$. Therefore in (2.11) we have that $C=\Pi^{\prime}\left(R_{i}^{\rho}(x)\right)+R_{i}^{H}(x)$, which proves the result setting $H_{\Delta x}(x)=R_{i}^{H}(x)$.

It is important to remark that, even if the reconstruction procedure satisfies the discrete steady state of the system in (2.11), the semidiscrete numerical scheme (2.10) may not be in general well-balanced. This is because the integral

$$
\int_{x_{i-1 / 2}}^{x_{i+1 / 2}} S_{H}\left(R_{i}^{U}(x), R_{i}^{H}(x)\right) d x
$$

has to be numerically approximated, and if such integration is not exact then the well-balancing property may be destroyed (see [26]). To overcome this difficulty, we follow the strategy proposed in [26]: a local discrete stationary solution is added to the numerical scheme for every cell. We denote this solution by $U_{i}^{*}(x)=\left(\rho_{i}^{*}(x), 0\right)^{T}$ and $H_{i}^{*}(x)$, and it satisfies

$$
\frac{1}{\Delta x}\left(F\left(U_{i}^{*}\left(x_{i+1 / 2}\right)\right)-F\left(U_{i}^{*}\left(x_{i-1 / 2}\right)\right)\right)=\frac{1}{\Delta x} \int_{x_{i-1 / 2}}^{x_{i+1 / 2}} S_{H}\left(U_{i}^{*}(x), H_{i}^{*}(x)\right) d x .
$$

The previous steady state relation in (2.15) is satisfied if we choose $U_{i}^{*}(x)=$ $\left(\rho_{i}^{*}(x), 0\right)^{T}$ and $H_{i}^{*}(x)$ as

$$
U_{i}^{*}(x)=\left(\begin{array}{c}
\rho_{i}^{*}(x)=R_{i}^{\rho}(x) \\
0
\end{array}\right), \quad H_{i}^{*}(x)=K_{i}-\Pi^{\prime}\left(R_{i}^{\rho}(x)\right) .
$$

Observe that the convolution is indirectly approximated in (2.16).

Now, we can rewrite the semidiscrete numerical scheme (2.10) by just adding the steady state expression in (2.15), yielding

$$
\begin{aligned}
\frac{d U_{i}}{d t}= & -\frac{1}{\Delta x}\left(\mathbb{F}_{i+1 / 2}^{-}-\mathbb{F}_{i-1 / 2}^{+}-F\left(U_{i}^{*}\left(x_{i+1 / 2}\right)\right)+F\left(U_{i}^{*}\left(x_{i-1 / 2}\right)\right)\right) \\
& +\frac{1}{\Delta x} \int_{x_{i-1 / 2}}^{x_{i+1 / 2}} S_{H}\left(R_{i}^{U}(x), R_{i}^{H}(x)\right)-S_{H}\left(U_{i}^{*}(x), H_{i}^{*}(x)\right) d x
\end{aligned}
$$

The advantage of this new version of the scheme relies on the fact that the integral term in (2.17) could be approximated by any high-order quadrature formula, without perturbing the well-balanced character of the numerical scheme. This comes from the fact that, for any discrete stationary solution satisfying (2.4), we have $R_{i}^{H}(x)=H_{i}^{*}(x)$ and $R_{i}^{\rho}(x)=\rho_{i}^{*}(x)$, so that

$$
S_{H}\left(R_{i}^{U}(x), R_{i}^{H}(x)\right)-S_{H}\left(U_{i}^{*}(x), H_{i}^{*}(x)\right)=0 .
$$

For such an integral we follow [68], where an $n$th order Richardson extrapolation formula is proposed to evaluate source terms of the form $\rho \partial_{x} H$. We detail the fourthand sixth-order formulas in Appendix SM2. We finally conclude with the following result.

THEOREM 2.3. The numerical scheme (2.17) with the reconstruction operators (2.13) and (2.14) is well-balanced in the sense of Definition 2.1.

Copyright $@$ by SIAM. Unauthorized reproduction of this article is prohibited. 
Proof. Let us assume that $u=0, \rho(x), H_{\Delta x}(x)$ satisfy (2.4). We also assume for simplicity that the stationary solution is defined on one connected component. Then, as proved in Theorem $(2.2)$, the reconstructions $R_{i}^{\rho}(x), R_{i}^{\rho u}(x)$, and $R_{i}^{H}(x)$ satisfy (2.11). Moreover,

$$
\frac{1}{\Delta x} \int_{x_{i-1 / 2}}^{x_{i+1 / 2}} S_{H}\left(R_{i}^{U}(x), R_{i}^{H}(x)\right)-S_{H}\left(U_{i}^{*}(x), H_{i}^{*}(x)\right) d x=0,
$$

and the reconstructed states at the intercells verify

$$
\Pi^{\prime}\left(\rho_{i+1 / 2}^{+}\right)+H_{i+1 / 2}^{+}=\Pi^{\prime}\left(\rho_{i+1 / 2}^{-}\right)+H_{i+1 / 2}^{-}=C, \quad u_{i+1 / 2}^{-}=u_{i+1 / 2}^{+}=0 .
$$

The relation (2.18) implies that the hydrostatic-reconstructed states satisfy

$$
\rho_{i+1 / 2}^{H R,-}=\rho_{i+1 / 2}^{H R,+}, \quad(\rho u)_{i+1 / 2}^{H R,--}=(\rho u)_{i+1 / 2}^{H R,+}=0 .
$$

Using (2.18) and (2.19) and the definition of (2.6) and (2.9), $\mathbb{F}_{i+1 / 2}^{-}$reduces to

$$
\mathbb{F}_{i+1 / 2}^{-}=\left(\begin{array}{c}
0 \\
P\left(\rho_{i+1 / 2}^{-}\right.
\end{array}\right) \text {. }
$$

Analogously, we deduce that

$$
\mathbb{F}_{i-1 / 2}^{+}=\left(\begin{array}{c}
0 \\
P\left(\rho_{i-1 / 2}^{+}\right)
\end{array}\right) \text {. }
$$

Now, taking into account the definition of $U_{i}^{*}(x)$ given in (2.16),

$$
U_{i}^{*}\left(x_{i+1 / 2}\right)=\left(\begin{array}{c}
\rho_{i+1 / 2}^{-} \\
0
\end{array}\right), \quad U_{i}^{*}\left(x_{i-1 / 2}\right)=\left(\begin{array}{c}
\rho_{i-1 / 2}^{+} \\
0
\end{array}\right),
$$

we finally conclude that the numerical scheme (2.17) reduces to

$$
\frac{d U_{i}}{d t}=\frac{1}{\Delta x}\left(\mathbb{F}_{i+1 / 2}^{-}-\mathbb{F}_{i-1 / 2}^{+}-F\left(U_{i}^{*}\left(x_{i+1 / 2}\right)\right)+F\left(U_{i}^{*}\left(x_{i-1 / 2}\right)\right)\right)=0 .
$$

Remark 2.4. The well-balanced reconstruction operators defined in (2.13) and (2.14) employ, as expected, the approximated cell averages of the solution at each time step and an extra quantity corresponding to the cell average of the variation of the free energy, denoted by $K_{i}$. This quantity plays an important role in achieving the well-balanced property of the reconstruction operators and the final numerical scheme. Note that the semidiscrete numerical scheme (2.17) allows only the cell averages of conserved variables to evolve in time and, as a result, we should provide an extra equation to evolve the variation of the free energy. We propose the following: suppose that $\left\{\rho_{i}^{n}\right\},\left\{(\rho u)_{i}^{n}\right\}$, and $\left\{K_{i}^{n}\right\}$ are known at time $t=n \Delta t$ and, suppose in addition, that we use the standard explicit first-order Euler scheme to evolve the conserved variables up to time $t=(n+1) \Delta t$. We then propose to update $K_{i}^{n+1}$ as

$$
\begin{aligned}
K_{i}^{n+1}= & K_{i}^{n}+\sum_{j=1}^{n_{s}} \alpha_{j}\left[\Pi^{\prime}\left(R_{i}^{\rho^{n+1}}\left(x_{i}^{j}\right)\right)-\Pi^{\prime}\left(R_{i}^{\rho^{n}}\left(x_{i}^{j}\right)\right)\right] \\
& +\sum_{j=1}^{n_{s}} \alpha_{j}\left[\sum_{l=1}^{M} \sum_{m=1}^{n_{s}} \Delta x \alpha_{m} W\left(x_{i}^{j}-x_{l}^{m}\right)\left[R_{l}^{\rho^{n+1}}\left(x_{l}^{m}\right)-R_{l}^{\rho^{n}}\left(x_{l}^{m}\right)\right]\right],
\end{aligned}
$$

Copyright $@$ by SIAM. Unauthorized reproduction of this article is prohibited. 
where we use some high-order quadrature formula for the cell averages and convolution operator. Here we will use the fifth-order Gaussian quadrature. A similar procedure could be applied if a high-order Runge-Kutta (RK)-TVD scheme (see [51]) is used instead of the explicit Euler scheme to discretize the ODE system (2.17). This is based on the classical observation that these schemes can be written as linear combinations of explicit Euler steps. Observe also that $K_{i}^{n+1}=K_{i}^{n}$ on discrete stationary solutions satisfying (2.4).

Finally, if the term $S_{D}(x, U)$ is now added to the system, our full high-order semidiscrete well-balanced finite volume scheme can be written as

$$
\begin{aligned}
\frac{d U_{i}}{d t}= & -\frac{1}{\Delta x}\left(\mathbb{F}_{i+1 / 2}^{-}-\mathbb{F}_{i-1 / 2}^{+}-F\left(U_{i}^{*}\left(x_{i+1 / 2}\right)\right)+F\left(U_{i}^{*}\left(x_{i-1 / 2}\right)\right)\right) \\
+ & \frac{1}{\Delta x} \int_{x_{i-1 / 2}}^{x_{i+1 / 2}} S_{H}\left(R_{i}^{U}(x), R_{i}^{H}(x)\right)-S_{H}\left(U_{i}^{*}(x), H_{i}^{*}(x)\right) d x \\
- & \gamma(\rho u)_{i}+\frac{1}{\Delta x} \sum_{j=1}^{n_{s}} \alpha_{j} R_{i}^{\rho, t}\left(x_{i}^{j}\right) \\
& \cdot\left[\sum_{l=1}^{M} \sum_{m=1}^{n_{s}} \Delta x \alpha_{m} \psi\left(x_{i}^{j}-x_{l}^{m}\right)\left(R_{i}^{u, t}\left(x_{i}^{j}\right)-R_{l}^{u, t}\left(x_{l}^{m}\right)\right) R_{l}^{\rho, t}\left(x_{l}^{m}\right)\right],
\end{aligned}
$$

where

$$
R_{i}^{u, t}=\frac{R_{i}^{\rho u, t}}{R_{i}^{\rho, t}} .
$$

Note that the new terms do not affect the well-balanced property of the scheme as they vanish when $u=0$.

Remark 2.5. In practical applications it is quite important to guarantee that the numerical scheme preserves the nonnegativity of the density $\rho_{i}(t)$. This is indeed the case with the numerical scheme (2.21) as a consequence of

1. the first order numerical flux preserves the nonnegativity of the density (see $[20])$;

2. the application of the positive-density limiter introduced into [82] and described in Appendix SM1.3;

3. a suitable CFL restriction (see (SM1.9) and [82]), described also in Appendix SM1.3.

2.4. Algorithmic implementation of the scheme. We now summarize the steps to efficiently implement the high-order well-balanced finite-volume scheme of subsection 2.3.

The initial conditions for system (2.1) are the initial density profile $\rho_{0}(x)$ and the initial momentum profile $(\rho u)_{0}(x)$. These initial conditions are introduced into the numerical scheme by computing their cell averages via the high-order quadrature formula,

$$
\begin{gathered}
\rho_{i}^{0}=\sum_{j=1}^{n_{s}} \alpha_{j} \rho_{0}\left(x_{i}^{j}\right), \\
(\rho u)_{i}^{0}=\sum_{j=1}^{n_{s}} \alpha_{j}(\rho u)_{0}\left(x_{i}^{j}\right),
\end{gathered}
$$

Copyright $\odot$ by SIAM. Unauthorized reproduction of this article is prohibited. 
where the coefficients $\alpha_{j}$ denote the weights of the quadrature formula that multiply the evaluation of $\rho_{0}(x)$ and $(\rho u)_{0}(x)$ at the quadrature points $x_{i}^{j}$, and $n_{s}$ denotes the number of quadrature points. Here we employ the fifth-order Gaussian quadrature formula.

The initial cell averages of the derivative of the free energy (2.4) are also required, and are similarly computed via fifth-order Gaussian quadrature

$$
K_{i}^{0}=\sum_{j=1}^{n_{s}} \alpha_{j}\left[\Pi^{\prime}\left(\rho_{0}\left(x_{i}^{j}\right)\right)+V\left(x_{i}^{j}\right)+\sum_{l=1}^{n} \sum_{m=1}^{n_{s}} \Delta x \alpha_{m} W\left(x_{i}^{j}-x_{l}^{m}\right) \rho_{0}\left(x_{l}^{m}\right)\right] .
$$

The computation of the cell averages using quadrature formulas is only necessary at the initial time step of the algorithm. For further time steps, the algorithm presented here takes as inputs the cell averages $\rho_{i}^{n},(\rho u)_{i}^{n}$, and $K_{i}^{n}$, evaluated at $t=n \Delta t$, and directly returns the cell averages $\rho_{i}^{n+1},(\rho u)_{i}^{n+1}$, and $K_{i}^{n+1}$ at the subsequent time step $t=(n+1) \Delta t$. The steps for such an algorithm are

(1) perform high-order reconstructions $R_{i}^{\rho}(x), R_{i}^{\rho u}(x)$, and $R_{i}^{K}(x)$ from the sequences of cell values $\left\{\rho_{i}^{n}\right\},\left\{(\rho u)_{i}^{n}\right\},\left\{K_{i}^{n}\right\}$, following (2.13). In our case, such reconstructions are conducted via third- and fifth-order CWENO reconstructions [11, 61, 62], details are given in Appendix SM1.

For simplicity, the evaluations of the previous reconstructions at the intercells at time $t=n \Delta t$ are denoted as

$$
\begin{array}{cl}
\rho_{i-1 / 2}^{+}=R_{i}^{\rho}\left(x_{i-1 / 2}\right), & \rho_{i+1 / 2}^{-}=R_{i}^{\rho}\left(x_{i+1 / 2}\right), \\
(\rho u)_{i-1 / 2}^{+}=R_{i}^{\rho u}\left(x_{i-1 / 2}\right), & (\rho u)_{i+1 / 2}^{-}=R_{i}^{\rho u}\left(x_{i+1 / 2}\right), \\
K_{i-1 / 2}^{+}=R_{i}^{K}\left(x_{i-1 / 2}\right), & K_{i+1 / 2}^{-}=R_{i}^{K}\left(x_{i+1 / 2}\right) .
\end{array}
$$

Furthermore, when required, the reconstruction of the velocity field $u$ is computed as

$$
R_{i}^{u}(x)=\frac{R_{i}^{\rho u}(x)}{R_{i}^{\rho}(x)}, \quad u_{i-1 / 2}^{+}=R_{i}^{u}\left(x_{i-1 / 2}\right), \quad u_{i+1 / 2}^{-}=R_{i}^{u}\left(x_{i+1 / 2}\right) .
$$

(2) Obtain the reconstruction $R_{i}^{H}(x)$ for $H_{\Delta x}(x)$ from (2.14), and their evaluations at the intercells as

$$
H_{i-1 / 2}^{+}=R_{i}^{H}\left(x_{i-1 / 2}\right), \quad H_{i+1 / 2}^{-}=R_{i}^{H}\left(x_{i+1 / 2}\right) .
$$

In general $H_{i+1 / 2}^{+} \neq H_{i+1 / 2}^{-}$. The average value between them is taken as

$$
H_{i+1 / 2}=\max \left(H_{i+1 / 2}^{+}, H_{i+1 / 2}^{-}\right) .
$$

(3) Reconstruct the local discrete stationary solution in (2.15) for every cell, so that $U_{i}^{*}(x)$ and $H_{i}^{*}(x)$ are computed from (2.16).

(4) Perform the so-called hydrostatic reconstruction described in (2.8), but now with the high-order reconstructions at the intercells. By denoting as $\xi(s)$ the 
inverse function of $\Pi^{\prime}(s)$ for $s>0$,

$$
\begin{array}{ll}
\rho_{i+1 / 2}^{H R,-}=\xi\left(\Pi^{\prime}\left(\rho_{i+1 / 2}^{-}\right)+H_{i+1 / 2}^{-}-H_{i+1 / 2}\right), & (\rho u)_{i+1 / 2}^{H R,-}=\rho_{i+1 / 2}^{H R,-} u_{i+1 / 2}^{-}, \\
\rho_{i+1 / 2}^{H R,+}=\xi\left(\Pi^{\prime}\left(\rho_{i+1 / 2}^{+}\right)+H_{i+1 / 2}^{+}-H_{i+1 / 2}\right), & (\rho u)_{i+1 / 2}^{H R,+}=\rho_{i+1 / 2}^{H R,+} u_{i+1 / 2}^{+} .
\end{array}
$$

(5) The cell averages $\rho_{i}^{n+1}$ and $(\rho u)_{i}^{n+1}$ at the subsequent time step $t=(n+1) \Delta t$ are updated by means of $(2.21)$, where

$$
\mathbb{F}_{i+1 / 2}^{ \pm}\left(U_{i+1 / 2}^{H R,-}, U_{i+1 / 2}^{H R,+}, H_{i+1 / 2}^{-}, H_{i+1 / 2}^{+}\right)=\mathcal{F}\left(U_{i+1 / 2}^{H R,-}, U_{i+1 / 2}^{H R,+}\right) \pm S_{i+1 / 2}^{H R, \pm}
$$

with the Lax-Friedrich flux in (2.7),

$$
\begin{aligned}
& S_{i+1 / 2}^{H R,+}=\left(\begin{array}{c}
0 \\
P\left(\rho_{i+1 / 2}^{+}\right)-P\left(\rho_{i+1 / 2}^{H R,+}\right)
\end{array}\right), \\
& \left.S_{i+1 / 2}^{H R,-}=\left(\begin{array}{c}
0 \\
P\left(\rho_{i+1 / 2}^{H R,-}\right)-P\left(\rho_{i+1 / 2}^{-}\right.
\end{array}\right)\right),
\end{aligned}
$$

$$
F\left(U_{i}^{*}\left(x_{i-1 / 2}\right)\right)=\left(\begin{array}{c}
0 \\
P\left(\rho_{i-1 / 2}^{+}\right)
\end{array}\right), \quad F\left(U_{i}^{*}\left(x_{i+1 / 2}\right)\right)=\left(\begin{array}{c}
0 \\
P\left(\rho_{i+1 / 2}^{-}\right)
\end{array}\right),
$$

and the integral for the high-order corrections in the source term is computed from the fourth- and sixth-order formulas in Appendix SM2.

(6) Finally, update the value $K_{i}^{n+1}$ by means of fluctuations from (2.20).

3. Numerical simulations. Here we employ the high-order finite volume scheme we developed in section 2 in a variety of relevant applications, taken from the fields of gas dynamics, porous media, collective behavior, and chemotaxis. First, in subsection 3.1 we confirm that the high-order and the well-balanced properties are numerically satisfied. Second, in subsection 3.2 we proceed to apply the scheme to challenging scenarios where analytical results are scarce.

The numerical flux for the simulations is chosen depending on the form of the pressure term, which satisfies $P(\rho)=\rho^{m}$ with $m \geq 1$. A local Lax-Friedrich numerical flux is employed for the examples with ideal-gas pressure, where $m=1$ and the support of the density is not compact. On the contrary, a kinetic scheme based on [71] is employed for pressures with $m>1$, due to the presence of vacuum regions and compactly supported densities. For the details of these two numerical fluxes we refer the reader to our previous work [20].

For the temporal integration we implement the third-order TVD RK method [51], with the CFL number chosen as 0.7 in all simulations. The CFL conditions for these two numerical fluxes are detailed in (SM1.9) of Appendix SM1. The boundary conditions are periodic unless otherwise specified. We set $\gamma=1$ in the linear damping while the nonlinear damping is in general deactivated, except for Example 3.4. The number of cells employed to create the plots is 200 . For the figures we use the thirdorder time discretization scheme, unless otherwise stated. 
We focus on the temporal evolution of the density, momentum, and free-energy variation in (2.12). For illustrative purposes, we also plot the evolution of the discrete versions of the total energy in (1.4) and free energy in (1.2), which are given by

$E^{\Delta}=\sum_{i} \frac{\Delta x_{i}}{2} \rho_{i} u_{i}^{2}+F^{\Delta} \quad$ and $\quad F^{\Delta}=\sum_{i} \Delta x_{i}\left[\Pi\left(\rho_{i}\right)+V_{i} \rho_{i}\right]+\frac{1}{2} \sum_{i, j} \Delta x_{i} \Delta x_{j} W_{i j} \rho_{i} \rho_{j}$.

It is worth mentioning that previous works have constructed finite-volume schemes that satisfy the discrete analog of the entropy identity in (1.6) (see "entropy stable schemes" in $[41,77]$ ) and free energy dissipation property in (1.5) (see "energy dissipating schemes" in $[3,12,20])$. The simulations do satisfy the discrete energy dissipation in practice, even if we are not able to prove it for our finite-volume scheme. The extension of the present scheme to satisfy the challenging discrete properties of entropy stability and energy dissipation with high-order accuracy will be explored elsewhere.

3.1. Validation of the numerical scheme. The validation of the finite-volume scheme in section 2 encompasses a test for the well-balanced property and a test for the high-order accuracy in the transient regimes. Both tests are conducted in two different scenarios, for which different choices of the free energy in (1.2) are taken.

On the one hand, for the well-balanced property we show that the steady state solution is preserved in time up to machine precision. For this we select as initial conditions a density and momentum profile satisfying the steady states obtained from (1.3). Numerically this means that the discrete version of the variation of the free energy in (2.4) holds, while the momentum vanishes throughout the domain. The results for this test are depicted in Table 3.1 for a simulation time run from $t=0$ to $t=5$ and a number of cells of 50 .

TABLE 3.1

Preservation of the steady state for Examples 3.1 and 3.2 with the third- and fifth-order schemes and machine precision in MATLAB, at $t=5$.

\begin{tabular}{lcc}
\hline & Order of the scheme & $L^{1}$ error \\
\hline \multirow{2}{*}{ Example 3.1} & 3rd & $1.7082 \mathrm{e}-16$ \\
& 5th & $1.7094 \mathrm{e}-16$ \\
\hline \multirow{2}{*}{ Example 3.2 } & 3rd & $5.5020 \mathrm{E}-17$ \\
& 5th & $6.4514 \mathrm{E}-17$ \\
\hline
\end{tabular}

On the other hand, the spatial order of accuracy in the transient regimes is computed by evaluating the $L^{1}$ error of the numerical solution for a particular mesh-size grid $\Delta x$ with respect to a reference solution. To measure the spatial high-order accuracy of the scheme, we select scenarios where shock waves or sharp gradients in the density and momentum profile do not evolve, such as the ones in Examples 3.1 and 3.2 , and we let the simulation run until $t=0.1$. We repeat this procedure by halving the $\Delta x$ from the previous simulation and the order of the scheme is computed as

$$
\text { order of the scheme }=\ln _{2}\left(\frac{L^{1} \operatorname{error}(\Delta x)}{L^{1} \operatorname{error}(\Delta x / 2)}\right) .
$$

For the system with a nonlocal free energy in (1.1) there are generally no explicit solutions in the transient regime. This implies that the reference solution has to be 
TABle 3.2 $t=0.1$.

Spatial order-of-convergence test for Example 3.1 with the third- and fifth-order schemes, at

\begin{tabular}{ccccc}
\hline \multirow{2}{*}{$\begin{array}{c}\text { Number of } \\
\text { cells }\end{array}$} & \multicolumn{2}{c}{ Third-order } & \multicolumn{2}{c}{ Fifth-order } \\
\cline { 2 - 5 }$L^{1}$ error & order & $L^{1}$ error & order \\
\hline 50 & $1.4718 \mathrm{E}-04$ & - & $1.9260 \mathrm{E}-05$ & - \\
\hline 100 & $2.3726 \mathrm{E}-05$ & 2.63 & $5.1254 \mathrm{E}-07$ & 5.23 \\
\hline 200 & $2.4182 \mathrm{E}-06$ & 3.29 & $2.1997 \mathrm{E}-08$ & 4.54 \\
\hline 400 & $2.6708 \mathrm{E}-07$ & 3.18 & $9.2613 \mathrm{E}-10$ & 4.57 \\
\hline
\end{tabular}

computed from the same numerical scheme using an extremely refined $\Delta x$ with the aim of accepting that numerical solution as the exact one. Here we take 25600 cells to compute such a reference solution, while the other numerical simulations for the order of accuracy employ 50,100, 200, and 400 cells. The results showing the spatial thirdand fifth-order of accuracy for the scenarios in Examples 3.1 and 3.2 are displayed in Tables 3.2 and 3.3. The time step has to be selected as $\Delta t \approx \Delta x$ and $\Delta t \approx(\Delta x)^{5 / 3}$ for the third- and fifth-order spatial discretizations, respectively, to obtain the correct order of convergence in space. This is because we employ a third-order TVD RK temporal integrator, a common practice in the literature, and we refer the reader to $[80$, subsection 5.4] as an example with the same choice for $\Delta t$.

Example 3.1 (ideal-gas pressure under an attractive potential). For this example we select an ideal-gas free energy with pressure $P(\rho)=\rho$ (or, equivalently, $\Pi(\rho)=$ $\rho \ln (\rho)-1)$ and with a quadratic external potential $V(x)=x^{2} / 2$. The steady state that we aim to preserve follows from

$$
\frac{\delta \mathcal{F}}{\delta \rho}=\Pi^{\prime}(\rho)+H(x, \rho)=\ln (\rho)+\frac{x^{2}}{2}=\text { constant on } \operatorname{supp}(\rho) \text { and } u=0 .
$$

Free energies of this type appear in the context of chemotaxis with a fixed chemoattractant profile [40, 43, 72], where cells will typically vary their direction when reacting to the presence of a chemical substance, so that they are attracted by chemically favorable environments and dodge unfavorable ones. In chemotaxis there is a chemoattractant function playing a role similar to the external potential $V(x)$, and in more complex models this chemoattractant function may even have its own evolution equation, typically a parabolic one. There are numerous well-balanced schemes for chemotaxis. Amongst them we highlight the fully implicit finite-volume scheme in [39], the scheme allowing for vacuum states in [67], the Godunov scheme in [50], and the high-order finite-volume and finite-differences schemes in [40, 66, 79].

The density profile for the steady state in (3.2) for an initial mass $M_{0}$ satisfies a Gaussian distribution of the form

$$
\rho_{\infty}=M_{0} \frac{e^{-x^{2} / 2}}{\int_{\mathbb{R}} e^{-x^{2} / 2} d x} .
$$

For the spatial order-of-accuracy test we take as initial condition a perturbation of the steady state in (3.3),

$$
\rho(x, t=0)=M_{0} \frac{e^{-x^{2} / 2}+0.1 * e^{-5(x+3)^{2}}}{\int_{\mathbb{R}}\left(e^{-x^{2} / 2}+0.1 * e^{-5(x+3)^{2}}\right) d x}, \quad \rho u(x, t=0)=0, \quad x \in[-5,5],
$$




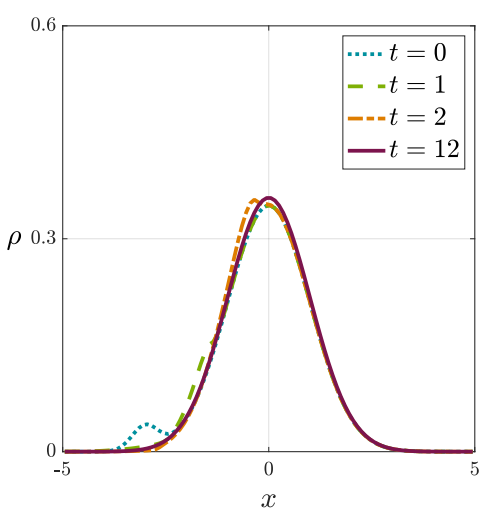

(a) Evolution of the density

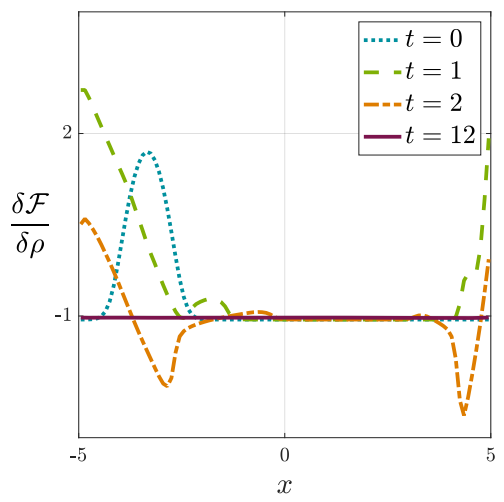

(c) Evolution of the variation of the free energy

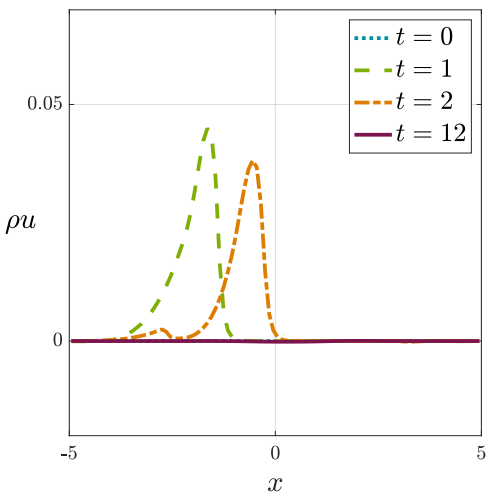

(b) Evolution of the momentum

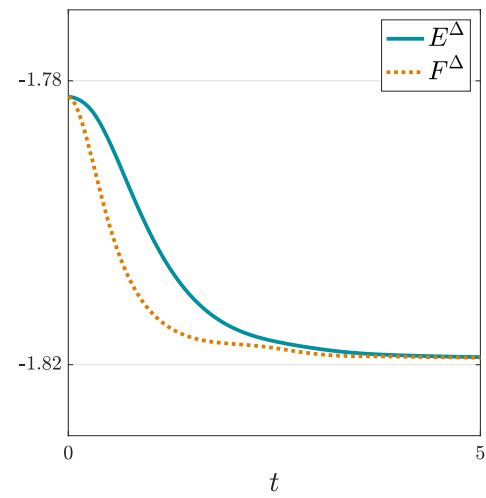

(d) Evolution of the total energy and the free energy

FIG. 3.1. Temporal evolution of Example 3.1.

with $M_{0}$ equal to 1 so that the total mass is also 1 . The spatial order-of-accuracy test from this example is shown in Table 3.2, while the temporal evolution of the density, momentum, free-energy with respect to the density, total energy, and free energy are displayed in Figure 3.1. The spatial third- and fifth-order of accuracies of the numerical scheme in section 2 are evident from Table 3.2. Figure 3.1(a) reveals that after $t=12$ the density converges to a Gaussian distribution corresponding to the steady state given in equation (3.3), while in Figure 3.1(c) the variation of the free energy is constant throughout the domain, given that the density is not compactly supported and (2.4) is satisfied. Finally, it is evident from Figure 3.1(d) that the discrete analogs of the total energy and free energy (3.1) decay in time.

In Figure 3.2 we visually illustrate the difference in accuracy between employing the third- and fifth-order schemes versus the first-order scheme in our previous work [20], which is summarized in subsection 2.1. We display the density and momentum fluctuation profiles at two different times $(t=0.2$ and $t=0.4)$, which result by substracting the initial conditions in (3.4) from the numerical profiles obtained with the same mesh of 100 cells for the three schemes. Having resolved the transient behavior, the difference between the various schemes can be elucidated by measuring 


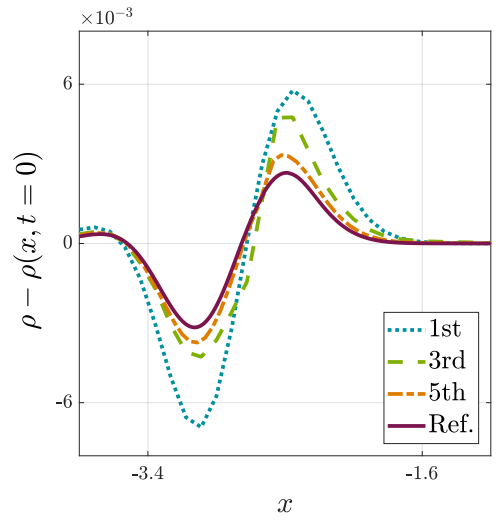

(a) Density fluctuations at $t=0.2$

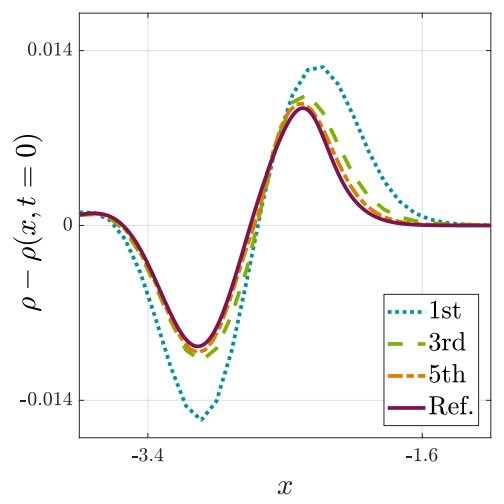

(c) Density fluctuations at $t=0.4$

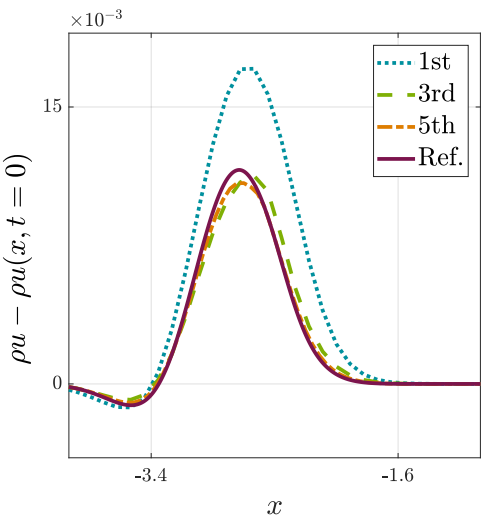

(b) Momentum fluctuations at $t=0.2$

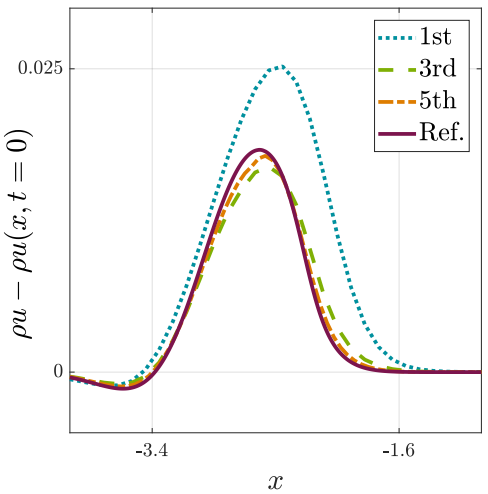

(d) Momentum fluctuations at $t=0.4$

FIG. 3.2. Density and momentum fluctuations in Example 3.1 for a first-, third-, and fifth-order scheme with the same mesh of 100 cells. The reference solution is computed with the third-order scheme and 12600 cells.

the fluctuations with respect to the initial condition. We also plot a reference profile obtained with the third-order scheme and 12600 cells. From Figure 3.2 we observe the benefit of employing the high-order schemes in comparison to the first-order one, since they provide a numerical solution much closer to the reference profile for the same number of cells.

Example 3.2 (generalized Euler-Poisson system: ideal-gas pressure and attractive kernel). For this example we select an ideal-gas free energy with pressure $P(\rho)=\rho$ (or, equivalently, $\Pi(\rho)=\rho \ln (\rho)-1)$ together with an interaction potential with a kernel of the form $W(x)=\frac{x^{2}}{2}$. In this case the steady state aimed to be preserved satisfies

$$
\frac{\delta \mathcal{F}}{\delta \rho}=\Pi^{\prime}(\rho)+H(x, \rho)=\ln (\rho)+\frac{x^{2}}{2} \star \rho=\text { constant on } \operatorname{supp}(\rho) \text { and } u=0 .
$$

Free energies of this type are common in Euler-Poisson systems, in which the Euler equations for a compressible gas are coupled to a self-consistent force field created by the gas particles [55]. This interaction could be gravitational, leading to the modeling of Newtonian stars [7], or electrostatic with repelling forces between the 
TABLE 3.3 $t=0.1$.

Spatial order-of-convergence test for Example 3.2 with the third-and fifth-order schemes, at

\begin{tabular}{ccccc}
\hline \multirow{2}{*}{$\begin{array}{c}\text { Number of } \\
\text { cells }\end{array}$} & \multicolumn{2}{c}{ Third-order } & \multicolumn{2}{c}{ Fifth-order } \\
\cline { 2 - 5 }$L^{1}$ error & order & $L^{1}$ error & order \\
\hline 50 & $5.0109 \mathrm{E}-04$ & - & $1.0913 \mathrm{E}-04$ & - \\
\hline 100 & $1.2721 \mathrm{E}-04$ & 1.98 & $5.0556 \mathrm{E}-06$ & 4.43 \\
\hline 200 & $1.7573 \mathrm{E}-05$ & 2.86 & $5.3713 \mathrm{E}-08$ & 6.56 \\
\hline 400 & $2.3001 \mathrm{E}-06$ & 2.93 & $2.3448 \mathrm{E}-10$ & 4.52 \\
\hline
\end{tabular}

particles in the case of plasma [34, 53]. For Euler-Poisson systems the free energy contains a function $S(t, x)$ which follows a Poisson-like equation, so that

$$
\frac{\delta \mathcal{F}}{\delta \rho}=\Pi^{\prime}(\rho)+S(t, x) \quad \text { and } \quad \partial_{x x} S(t, x)=c \rho,
$$

with $c$ being either 1 for the gravitational case or -1 for the plasma one. The Poisson equation for $S(t, x)$ can be solved considering the fundamental solution of the Laplacian in one dimension [60], which leads to $2 S=-c|x| \star \rho$. Then, by plugging this expression for $S$ into the variation of the free energy in (3.6), one recovers the interaction potential $W(x)$ which is convoluted with the density $\rho$. For an $S(t, x)$ following the Poisson equation, the interaction potential is $W(x)=-c|x|$, but for $c=-1$ one can generalize it to a homogeneous kernel $W(x)=|x|^{\alpha} / \alpha$, where $\alpha>-1$ and $W(x)=\ln |x|$ when $\alpha=0$ for convention. A popular application of these more general kernels $W(x)$ is in the Keller-Segel system for cells and bacteria $[6,9,18]$ which we explore in Example 3.5.

For Example 3.2 we select $\alpha=2$, leading to the interaction potential $W(x)=\frac{x^{2}}{2}$ in the variation of the free energy in (3.5). The steady state for a general mass $M_{0}$ is equal to the steady state for Example 3.1 and satisfies (3.3). Notice that the particular choice of $W(x)=\frac{x^{2}}{2}$ and a symmetric initial condition makes this example analytically equivalent to the case of external quadratic potential in Example 3.1 with the same initial data; to see this, simply expand the convolution and use the reflection symmetry of the kernel. However, by treating it numerically as a convolution we are able to check the order of accuracy for interaction potentials. For the spatial order-of-accuracy test the initial condition is a symmetric perturbation of the steady state in (3.3),

$$
\begin{aligned}
& \rho(x, t=0) \\
& \quad=M_{0} \frac{e^{-x^{2} / 2}+0.05 * e^{-5(x+3)^{2}}+0.05 * e^{-5(x-3)^{2}}}{\int_{\mathbb{R}}\left(e^{-x^{2} / 2}+0.05 * e^{-5(x+3)^{2}}+0.05 * e^{-5(x-3)^{2}}\right) d x}, \quad \rho u(x, t=0)=0,
\end{aligned}
$$

with $x \in[-10,10]$ and $M_{0}$ equal to 1 so that the total mass is also 1 . The spatial order-of-accuracy test from this example is shown in Table 3.3, while the temporal evolution of the density, momentum, variation of the free energy with respect to the density, total energy, and free energy are depicted in Figure 3.3. The spatial thirdand fifth-order accuracies of the numerical scheme in section 2 are evident from Table 3.3. Figure 3.3(a) shows that the density remains symmetric at all times eventually reaching the steady state profile in (3.3). It is also evident from Figure 3.3(c) that the variation of free energy reaches a constant value in the regions where the density is noncompactly supported, while Figure 3.3(d) demonstrates that the total energy and free energy exhibit a temporal decay. 


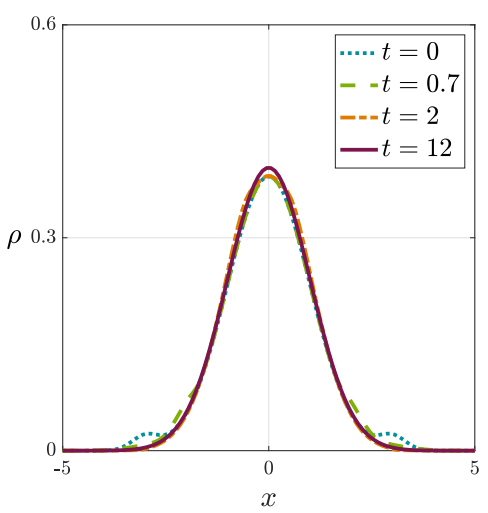

(a) Evolution of the density

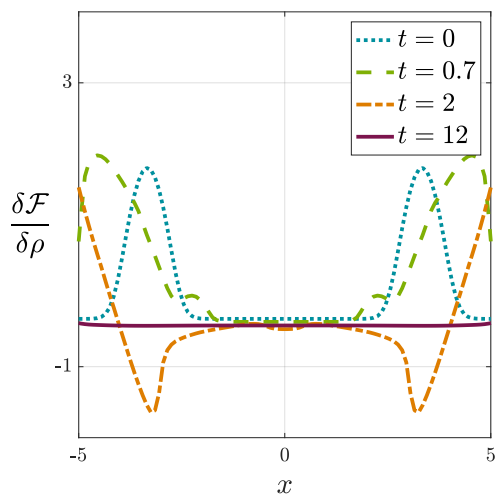

(c) Evolution of the variation of the free energy

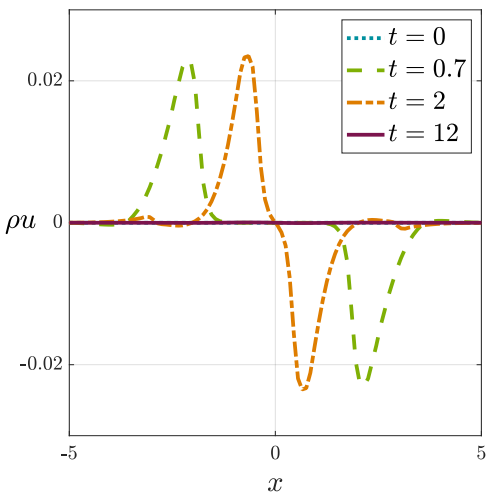

(b) Evolution of the momentum

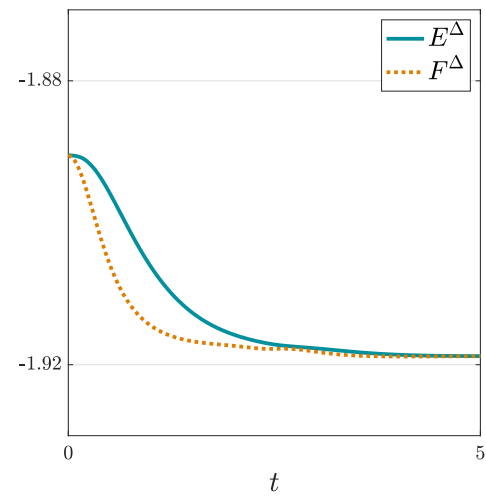

(d) Evolution of the total energy and the free energy

FIG. 3.3. Temporal evolution of Example 3.2.

3.2. Numerical experiments and applications. Here we apply the finitevolume scheme we developed in section 2 to applications of the shallow-water system, a collective behavior system with Cucker-Smale and Motsch-Tadmor damping terms, and the Keller-Segel model. Our scheme is useful to run challenging numerical experiments for which analytical results are limited in the literature, such as in the above applications.

Example 3.3 (shallow water: pressure proportional to square of density and attractive potential). In this example we select a pressure satisfying $P=\rho^{2}$ (or equivalently $\left.\Pi(\rho)=\rho^{2}\right)$ together with an attractive external potential $V(x)$. This scenario corresponds to the well-known shallow-water equations, which model free-surface gravity waves whose wavelength is much larger than the characteristic bottom depth. The choice $P=\rho^{2}$ leads to the presence of dry regions during the water-height evolution. These equations are applied to a wide range of engineering and scientific applications involving free-surface flows [81], such as tsunami propagation [27], dam break, and flooding problems [35] and the evolution of rivers and coastal areas [33].

The main three challenges to accurately simulate the shallow-water equations are the preservation of the steady states, the preservation of the water-height pos- 
itivity, and the transitions between wet and dry areas. Many authors have consequently proposed various numerical schemes addressing these challenges, employing methodologies ranging from finite-difference and finite-volume schemes to discontinuous Galerkin ones. The reader can find more relevant references about high-order schemes [24], well-balanced reconstructions [1], density positivity [82], and the simulation of the wet/dry front [42] in the introduction of this work and in the comprehensive survey from Xing and Shu [81].

For this example we aim to show that our numerical scheme accurately captures the dry regions during the simulation and when reaching the steady states. This is thanks to the combination of the positive-density reconstruction in Appendix SM1 and the choice of a kinetic numerical flux which is able to handle vacuum regions [71]. We show this by conducting simulations with two different choices for the external potential $V(x)$ with the following initial conditions for both cases:

$$
\rho(x, t=0)=\frac{e^{-\left(x-x_{0}\right)^{2} / 16}}{\int_{\mathbb{R}} e^{-\left(x-x_{0}\right)^{2} / 16} d x}, \quad \rho u(x, t=0)=-0.1 \sin \left(\frac{\pi x}{10}\right), \quad x \in[-5,5],
$$

with $x_{0}$ being the initial center of mass. The steady states for the choice of pressure and external potentials of this example satisfy

$$
\rho_{\infty}=(C(x)-V(x))_{+},
$$

where $C(x)$ is a piecewise constant function being zero outside the support of the density.

The details of each simulation are:

(1) Single-well external potential and symmetric density: $V(x)=x^{2} / 2$ and $x_{0}=$ 0 . The results of this simulation are depicted in Figure 3.4. Figure 3.4(a) shows the formation of a compact support of the density during the time evolution with the steady state taking the shape of a positive parabola and satisfying (3.7). We also observe that the variation of the free energy in Figure 3.4(c) reaches a constant value only in the support of the density, in agreement with the steady state relation in (1.3). We further note that in Figure 3.4(d) the discrete total energy decreases in time, while the discrete free energy has a slight increase around $t=3$ due to an exchange of energy with the kinetic energy.

(2) Double-well external potential and asymmetric density: $V(x)=x^{4} / 4-3 x^{2} / 2$ and $x_{0}=1.5$. The results of this simulation are depicted in Figure 3.5. From the evolution of the density in Figure 3.5(a) it is evident that two compactly supported bumps of density are formed when reaching the steady state. This is due to the external potential having two wells. In addition, the mass in the bumps is not the same, since the initial density is not symmetric. It is also important to remark that, when reaching the steady state, the variation of the free energy in each density bump is constant but has different values. This is depicted in Figure 3.5(c) and agrees with the steady state relation (1.3). We refer the reader to our previous work [20] for similar simulations considering varied scenarios with double-well potentials.

Example 3.4 (collective behavior: comparison of linear, Cucker-Smale, and Motsch-Tadmor dampings). In this example we explore the impact of adding linear and nonlinear damping terms to the general system (1.1). The motivation for 


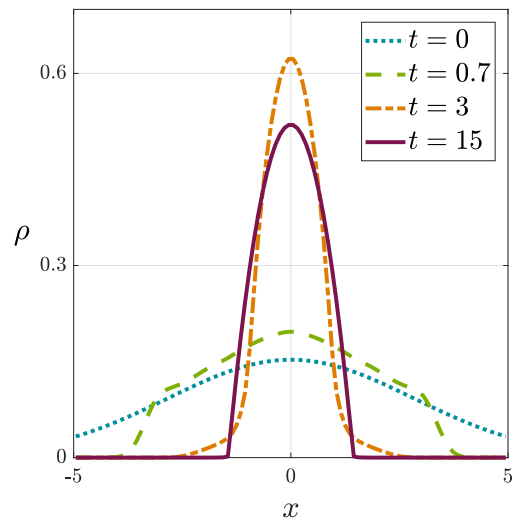

(a) Evolution of the density

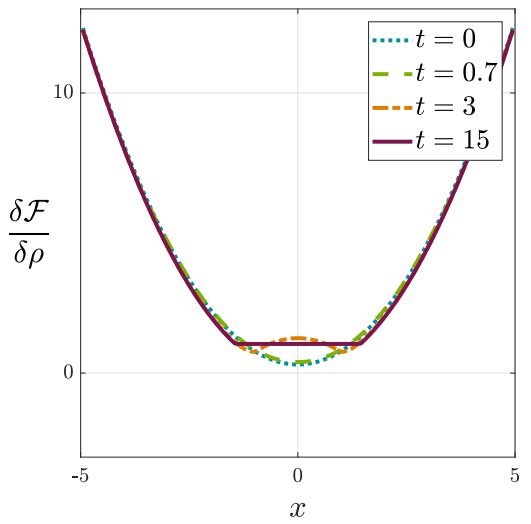

(c) Evolution of the variation of the free energy

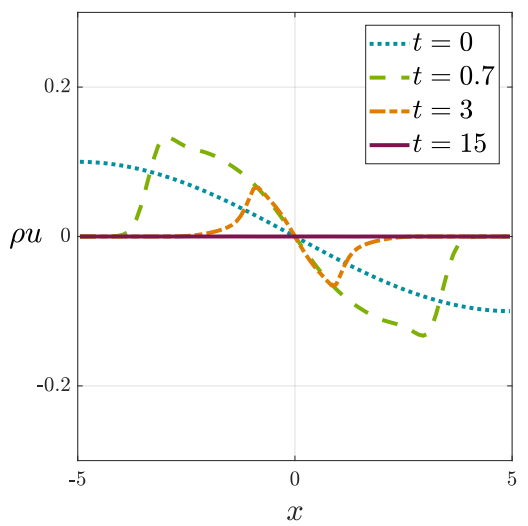

(b) Evolution of the momentum

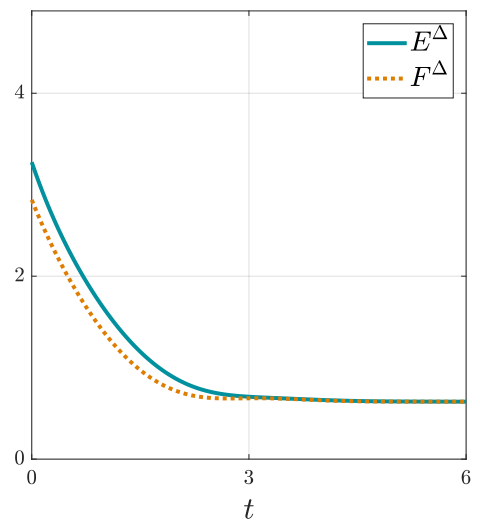

(d) Evolution of the total energy and the free energy

FIG. 3.4. Temporal evolution with single-well external potential and symmetric density in Example 3.3 .

the nonlinear damping comes from the field of collective behavior, in which a large amount of interacting individuals or agents organize their dynamics by influencing each other and without the presence of a leader. Most of the literature in collective behavior is based on agent models which are particle descriptions considering the three basic effects of attraction, repulsion, and alignment of the individuals. The combination of these three effects has proven to be very versatile and extends beyond the typical animal applications for schools of fish [57], herds of mammals [45], or flocks or birds [56]. Indeed, these models are now playing a critical role in understanding complex phenomena including consensus and spatio-temporal patterns in diverse problems ranging from the evolution of human languages [38] and the prediction of criminal behavior [73] to space flight formation [70].

There are plenty of works in the literature addressing the mean-field derivation of kinetic and other macroscopic models from the original particle descriptions $[16,17$, 54]. These derived hydrodynamic equations agree with our general system (1.1) and model the attraction and repulsion effects via the interaction potential $W(x)$. The third effect for collective behavior is alignment which in our system (1.1) is achieved by 


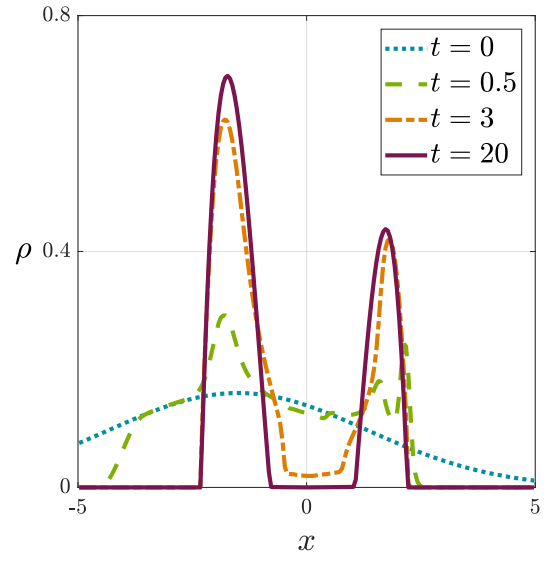

(a) Evolution of the density

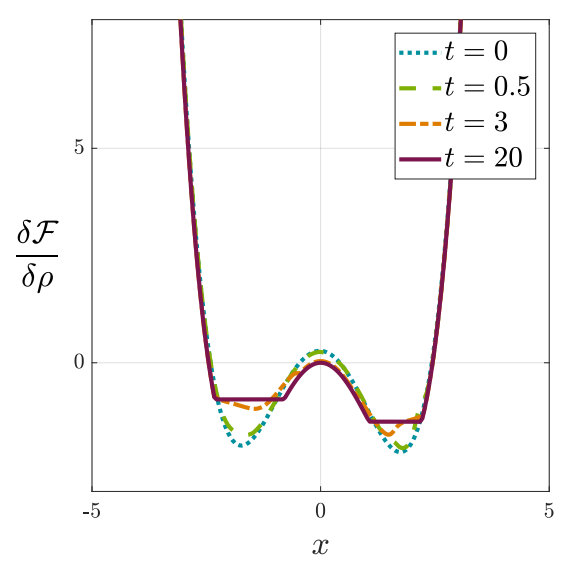

(c) Evolution of the variation of the free energy

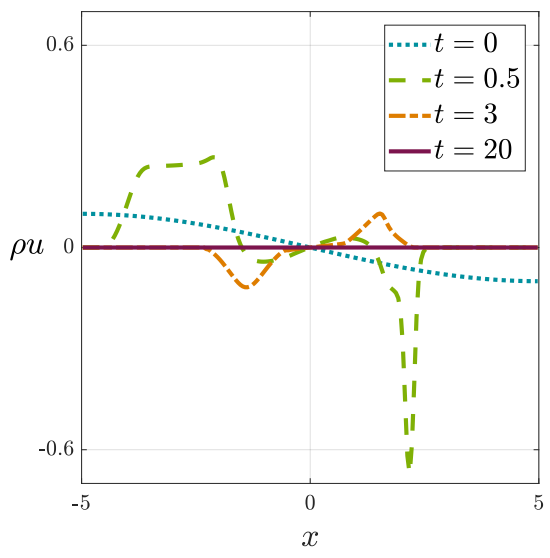

(b) Evolution of the momentum

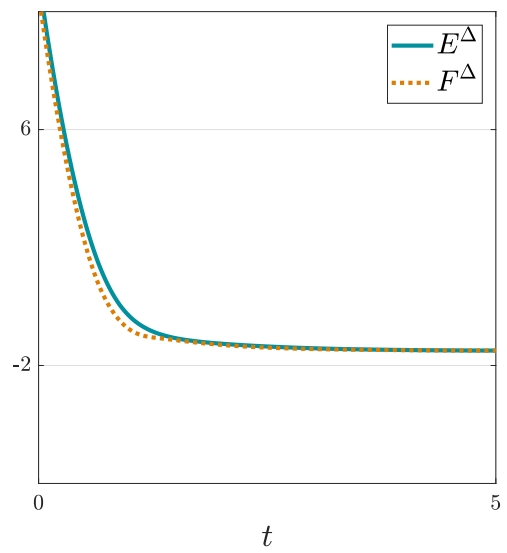

(d) Evolution of the total energy and the free energy

FIG. 3.5. Temporal evolution with double-well external potential and asymmetric density in Example 3.3.

means of the nonlinear and nonlocal damping of the right-hand side of the momentum equation. The most popular approach for the velocity consensus is the Cucker-Smale (CS) model $[36,37]$ which adapts the momentum of a particle depending on the momentum and distance of the other particles. Several authors have proposed refined variations of the CS model, and among them we note the weighted-normalized model by Motsch and Tadmor (MT) [65]. It basically corrects the CS model by eliminating the normalization over the total number of agents, which leads to inaccurate behavior in far-from-equilibrium scenarios. Instead, the MT model introduces the concept of relative distances between agents with the cost, however, of destroying the symmetry of the original CS model. For further details on flocking and alignment with the CS and related models, we refer the reader to [13, 14, 32, 64].

The objective of this example is to illustrate the differences of adding to the general system (1.1) linear damping, the CS or the MT model. The damping term 
for each of them is

$$
\begin{cases}-\gamma \rho \boldsymbol{u} & \text { if linear damping, } \\ -\rho \int_{\mathbb{R}^{d}} \psi(\boldsymbol{x}-\boldsymbol{y})(\boldsymbol{u}(\boldsymbol{x})-\boldsymbol{u}(\boldsymbol{y})) \rho(\boldsymbol{y}) d \boldsymbol{y} & \text { if CS damping, } \\ -\frac{\rho}{\psi \star \rho} \int_{\mathbb{R}^{d}} \psi(\boldsymbol{x}-\boldsymbol{y})(\boldsymbol{u}(\boldsymbol{x})-\boldsymbol{u}(\boldsymbol{y})) \rho(\boldsymbol{y}) d \boldsymbol{y} & \text { if MT damping, }\end{cases}
$$

where $\psi(x)$ is a nonnegative symmetric smooth function, called the communication function, satisfying for this example

$$
\psi(x)=\frac{1}{\left(1+|x|^{2}\right)^{\frac{1}{4}}} .
$$

It should be noted that the CS damping term in (3.8) would reduce to linear damping if the communication function $\psi(x)$ was a constant function $\psi(x)=1$. In addition, the difference between the CS and MT models is the normalization over $\psi \star \rho$ that is added to the MT model to ensure that the damping term is independent of the total mass of the system.

The simulation for this example is chosen to specifically address a particular drawback of the CS model. This occurs in the evolution of two groups of agents separated by a certain significant distance and whose masses have different degrees of magnitude. What happens with the CS model is that the damping term for the small group of agents is negligible due to the normalization over the total number of agents in the system. This means that small group agents do not seek alignment from the beginning of the simulation, and as a result convergence towards alignment is delayed. On the contrary, with the MT model the normalization over $\psi \star \rho$ in (3.8) allows us to take the relative distances between the agents into account, and the small group of agents reacts much faster to the effect of the rest of agents. In the simulation we also add a Morse-like interaction potential $[12,19]$ of the form $W(x)=-e^{-|x|^{2} / 2} / \sqrt{2 \pi}$, which quickly decays at large distances and does not add any attraction between the two groups of agents. Note that we are forced to add this attraction term to balance the pressure and thus allow for our well-balanced scheme in section 2. Finally, the pressure follows $P(\rho)=\rho$ (or, equivalently, $\Pi(\rho)=\rho \ln (\rho)-1$ ).

This configuration is depicted in Figure 3.6. Specifically, Figures 3.6(a) and (b) show the initial conditions for the density and the momentum. On the one hand, in the density there are two groups of agents with mass of 0.9 and 0.1 , satisfying

$$
\rho(x, t=0)=0.9 \frac{e^{-(x+1)^{2} / 2}}{\int_{\mathbb{R}} e^{-(x+1)^{2} / 2} d x}+0.1 \frac{e^{-(x-11)^{2}}}{\int_{\mathbb{R}} e^{-(x-11)^{2}} d x}, \quad x \in[-5,14],
$$

while on the other hand for the momentum the groups have opposite velocity signs, according to

$$
\rho u(x, t=0)=\left\{\begin{aligned}
2 \rho(x, t=0) & \text { if } x<5 \\
-2 \rho(x, t=0) & \text { if } x \geq 5
\end{aligned}\right.
$$




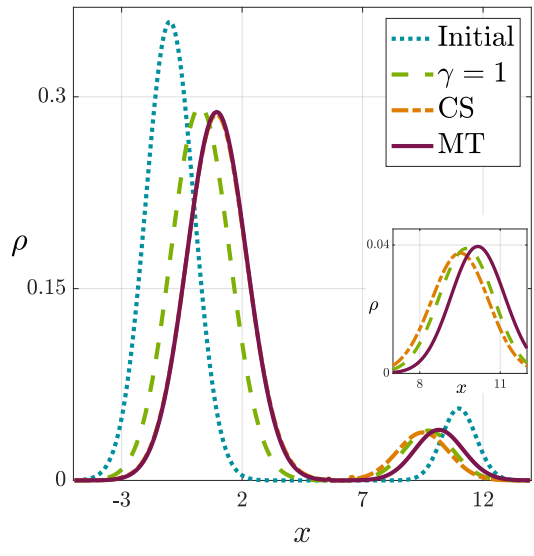

(a) Evolution of the density

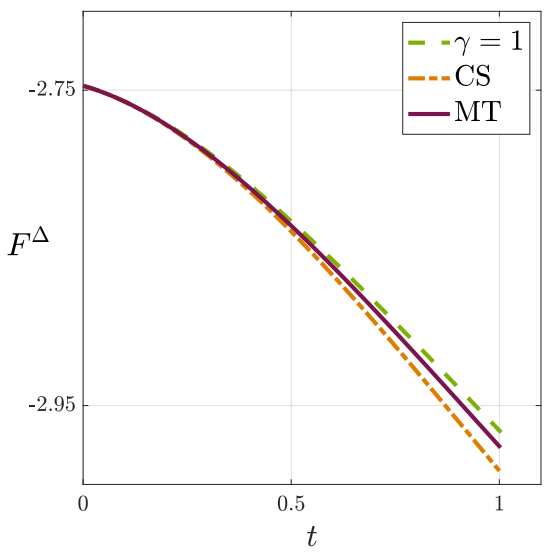

(c) Evolution of the discrete free energy

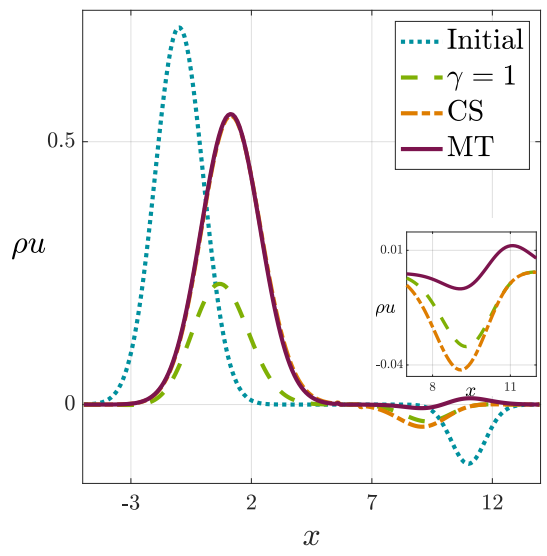

(b) Evolution of the momentum

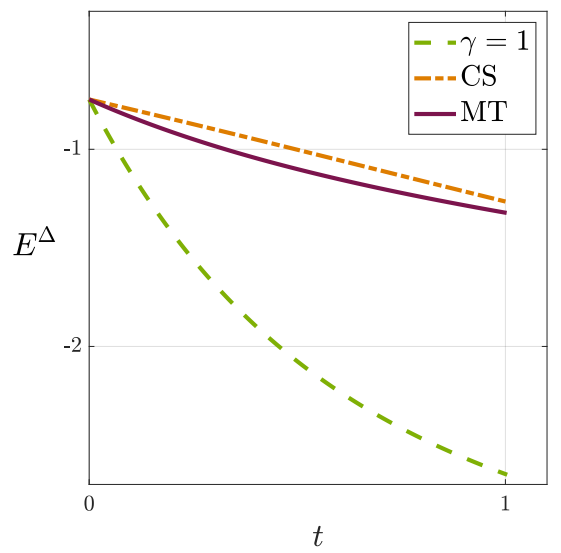

(d) Evolution of the discrete total energy

FIG. 3.6. Simulation of Example 3.4 until $t=1 . \gamma=1$ denotes the linear damping simulation, along with CS and MT simulations.

What we expect to happen in this situation is that the large group imposes its velocity sign over the small group, so eventually all the agents align with each other having positive velocity. From the momentum simulation in Figure 3.6(b) we observe that after $t=1$ the MT model has already changed the velocity sign of the small group from negative to positive, while for the CS model the velocity is still negative. In general, linear damping is the one that dissipates more momentum, as depicted in the momentum plot of Figure 3.6(b) and in the total energy plot of Figure 3.6(d). The free energy and total energy decay are comparable for both the CS and MT models. A similar numerical experiment was already conducted in [13] using particle methods.

Example 3.5 (hydrodynamic Keller-Segel system). The Keller-Segel model has been widely employed for chemotactic aggregation of biological populations such as cells, bacteria, or insects. It models how the production of a particular chemical by these organisms leads to long-range attraction and eventually results in selforganization. Its first formulation was proposed in [58] and consisted in a drift- 
diffusion equation for the density (which is obtained in the overdamped limit of our system (1.1)) coupled with a diffusion equation for the chemical concentration.

In this example we are interested in the hydrodynamic extension of the KellerSegel model proposed in [29] and take into account the inertia of the biological entities. It follows the same structure as the generalized Euler-Poisson system in Example 3.2 with the free energy satisfying (3.6) and the chemical concentration usually taken as $S=W(x) \star \rho$ (see [10, 18]). The homogeneous kernel $W(x)$ follows $W(x)=$ $|x|^{\alpha} / \alpha$, where $\alpha>-1$ and $W(x)=\ln |x|$ when $\alpha=0$ for convention. The difference with Example 3.2 is that here the pressure follows $P(\rho)=\rho^{m}$ with $m \geq 1$ (or, equivalently, $\left.\Pi(\rho)=\rho^{m} /(m-1)\right)$, thus allowing for compactly supported steady states and vacuum in the density if $m>1$. We refer the reader to [4] for more information about the Keller-Segel model and the diffusion equation for the chemical concentration.

In our previous work [20] we applied our first- and second-order well-balanced scheme to investigate the competition between the attraction from the local kernel $W(x)$ and the repulsion caused by the diffusion of the pressure $P(\rho)$. For the overdamped Keller-Segel model there are basically three possible regimes [9, 10], which result from adequately tuning the parameters $\alpha$ in the kernel $W(x)$ and $m$ in the pressure $P(\rho)$ : diffusion-dominated regime $(m>1-\alpha)$, balanced regime $(m=1-\alpha)$ where a critical mass separates self-similar and blowup behavior, and aggregationdominated regime $(m<1-\alpha)$. Results with the momentum equation, and thus inertia included, are still quite limited in the literature, with only some specific scenarios studied [14, 21]. In our previous work [20] we investigated the role of inertia for a choice of parameters of $\alpha=0.5, m=1.5$ and $\alpha=-0.5, m=1.3$, which led to diffusion- and aggregation-dominated regimes, respectively.

For this example we aim to explore the case $\alpha=0$ which leads to the singular potential $W(x)=\ln |x|$. Initially, for the two first simulations of this example we set $m=1$ so that $P(\rho)=\rho$. In the overdamped limit this scenario corresponds to the balanced regime since $m=1-\alpha$, and there is a critical mass separating the global-in-time from the finite-time blowup solutions. We run two simulations with identical initial conditions which differ only in a multiplicative constant for the density which allows us to set a different mass of the system. The objective is to determine how the mass of the system affects the solution. We set the initial conditions as

$$
\rho(x, t=0)=M \frac{e^{-(x)^{2} / 16}}{\int_{\mathbb{R}} e^{-(x)^{2} / 16} d x}, \quad \rho u(x, t=0)=0, \quad x \in[-8,8],
$$

with $M$ being the mass of the system.

For the first simulation we set the mass at $M=0.1$. The results are shown in Figure 3.7 where the numerical solution is clearly global-in-time and diffusion-dominated. Eventually the steady state is reached,

$$
\frac{\delta \mathcal{F}}{\delta \rho}=\Pi^{\prime}(\rho)+H(x, \rho)=\ln (\rho)+\ln |x| \star \rho=\text { constant on } \operatorname{supp}(\rho) \text { and } u=0 .
$$

From Figure 3.7(a) we observe that the solution is completely diffused and the final profile for the density is uniform. From Figure 3.7(c) we notice that the variation of 


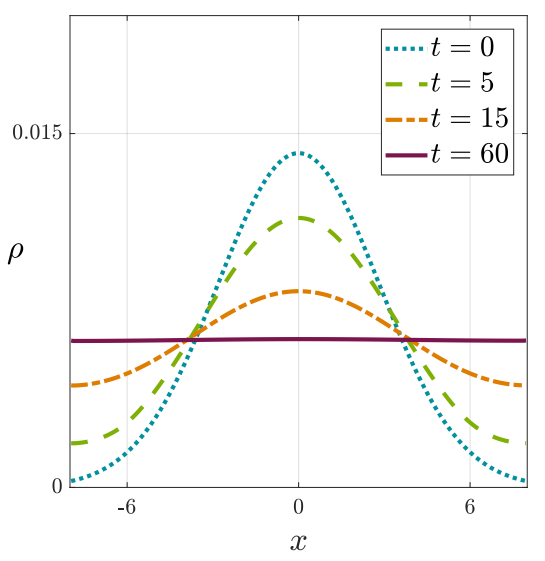

(a) Evolution of the density

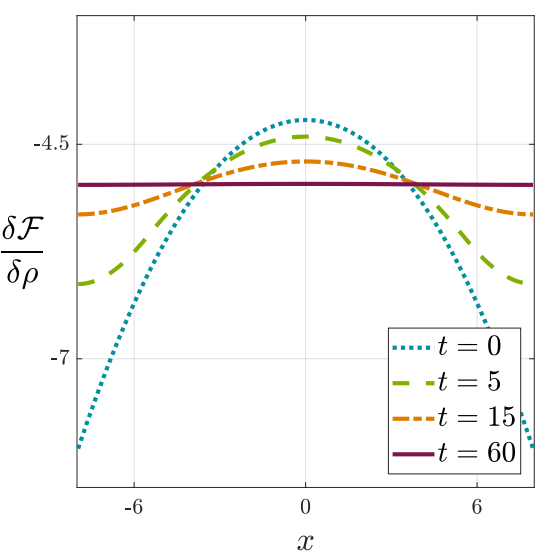

(c) Evolution of the variation of the free energy

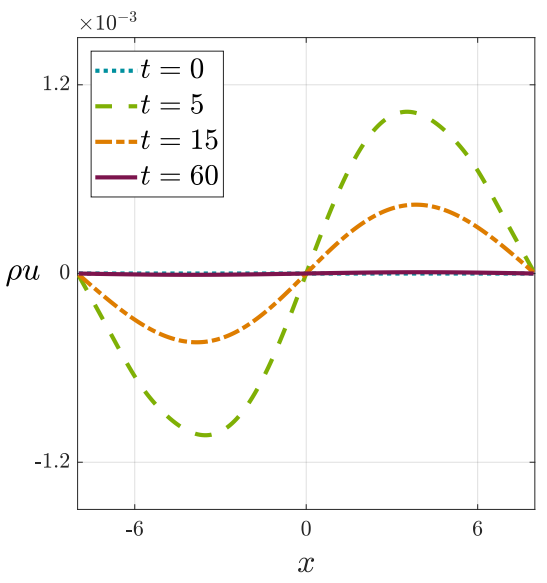

(b) Evolution of the momentum

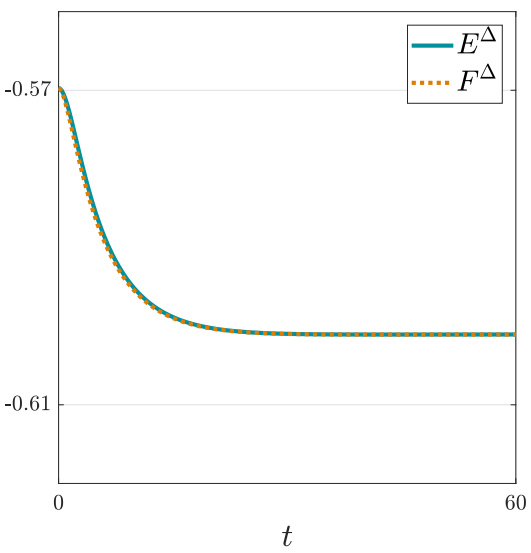

(d) Evolution of the total energy and the free energy

FIG. 3.7. Temporal evolution of the Keller-Segel system with $P=\rho, W(x)=\ln |x|$, and initial conditions (3.9) with $M=0.1$. The solution is global-in-time.

the free energy with respect to the density is constant once the steady state is reached, and from Figure 3.7(d) we observe how the total and free energy decay during the temporal evolution.

For the second simulation we select a mass of $M=3$ while keeping the same initial conditions as in (3.9). As displayed in Figure 3.8, the solution now presents a finite-time blowup around $t \approx 7.5$, leading to an aggregation-dominated behavior. Figure 3.8(a) reveals that the density is concentrated towards the middle of the domain while Figure 3.8(b) shows that the momentum develops a discontinuity with infinite slope at the origin when the density blows up. From Figure 3.8(d) we notice that the total and free energy temporally decay until the blowup occurs.

Finally, we also aim to compare diffusion-dominated solutions where $m>1$ leading to steady states that are compactly supported. For this purpose we set the initial conditions to be (3.9) with a mass $M=1$. For comparison we look at two scenarios with $P=3 \rho^{2}$ and $P=3 \rho^{2.5}$ so that the exponent $m$ is different. Figure 3.9 depicts 


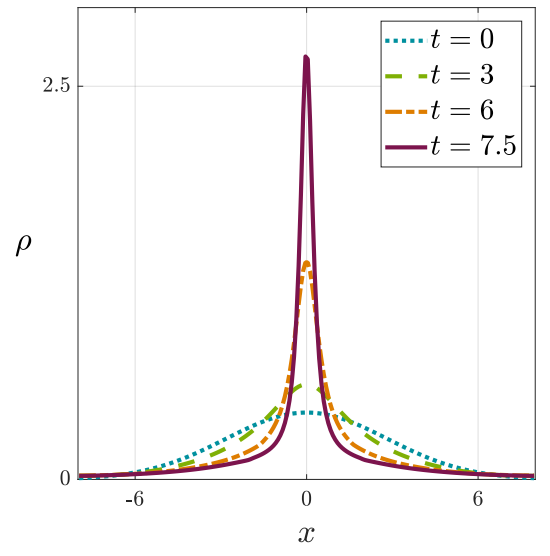

(a) Evolution of the density

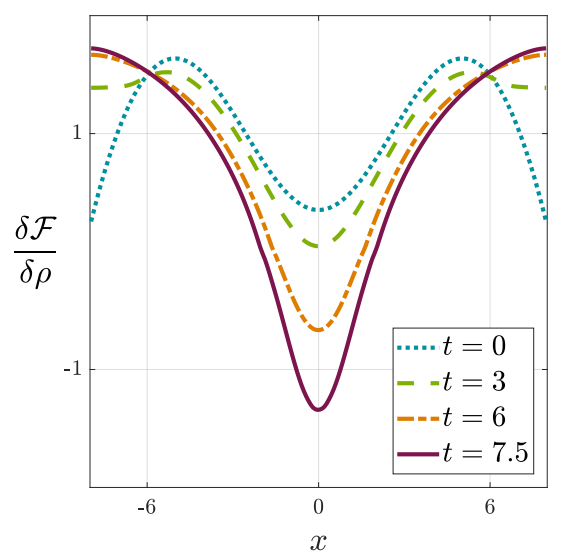

(c) Evolution of the variation of the free energy

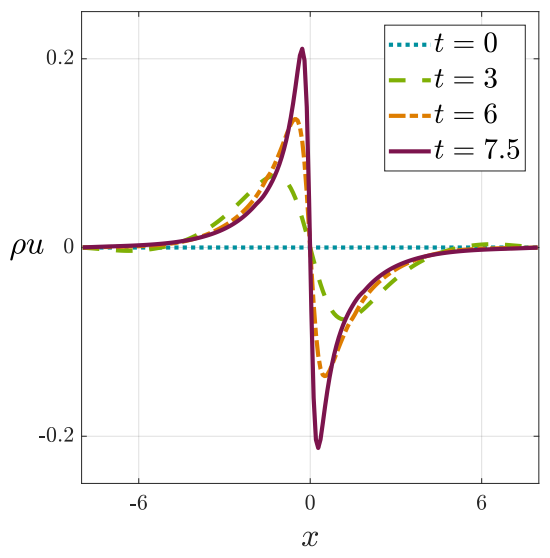

(b) Evolution of the momentum

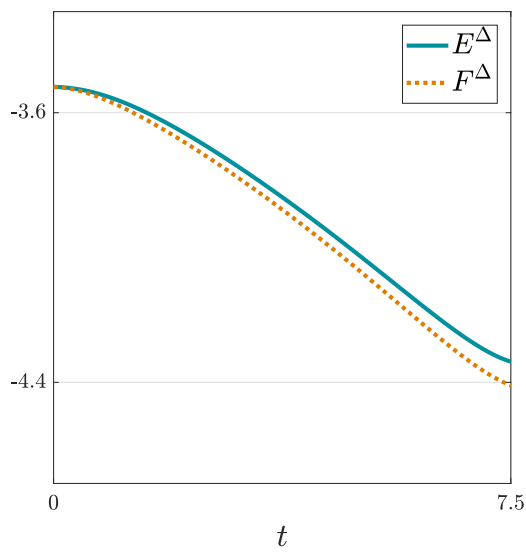

(d) Evolution of the total energy and the free energy

FIG. 3.8. Temporal evolution of the Keller-Segel system with $P=\rho, W(x)=\ln |x|$, and initial conditions (3.9) with $M=3$. The solution blows up in finite time.

the final steady states that arise from the two choices of $m$. From Figures 3.9(a) and (c) we observe that the final compactly supported density profiles have slightly different shapes due to the balances between the attraction from the local kernel $W(x)$ and the repulsion caused by the diffusion of the pressure $P(\rho)$.

Copyright (c) by SIAM. Unauthorized reproduction of this article is prohibited. 


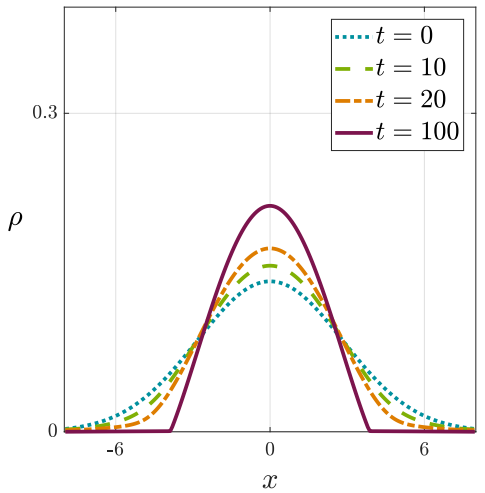

(a) Evolution of the density

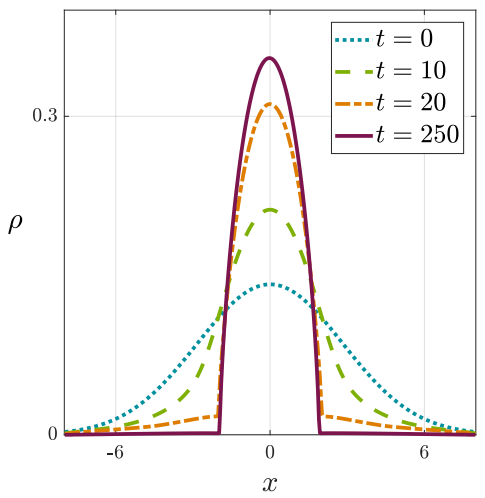

(c) Evolution of the density

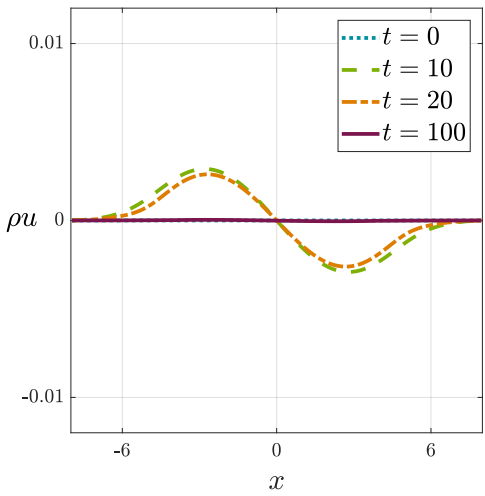

(b) Evolution of the momentum

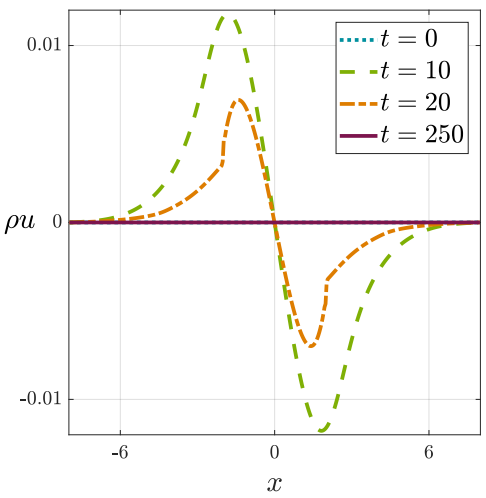

(d) Evolution of the total energy and the free momentum

FIG. 3.9. Temporal evolution of the Keller-Segel system with $W(x)=\ln |x|$ and initial conditions (3.9) with $M=1$. Compactly supported steady state. (a)-(b) $P=3 \rho^{2}$, steady state $t=250$; (c) - (d) $P=3 \rho^{2.5}$, steady state $t=100$.

Acknowledgment. Sergio P. Perez thanks Universidad de Málaga for hospitality during a visit in October 2019.

\section{REFERENCES}

[1] E. Audusse, F. Bouchut, M.-O. Bristeau, R. Klein, and B. Perthame, A fast and stable well-balanced scheme with hydrostatic reconstruction for shallow water flows, SIAM J. Sci. Comput., 25 (2004), pp. 2050-2065.

[2] E. Audusse, C. Chalons, And P. Ung, A very simple well-balanced positive and entropysatisfying scheme for the shallow-water equations, Commun. Math. Sci, 13 (2015), pp. 1317-1332.

[3] R. BAilo, J. A. CARrillo, And J. Hu, Fully discrete positivity-preserving and energy-decaying schemes for aggregation-diffusion equations with a gradient flow structure, Commun. Math. Sci., 18 (2020), pp. 1259-1303.

[4] N. Bellomo, A. Bellouquid, Y. TaO, and M. Winkler, Toward a mathematical theory of Keller-Segel models of pattern formation in biological tissues, Math. Models Methods Appl. Sci., 25 (2015), pp. 1663-1763.

[5] A. Bermúdez AND M. E. VÁzQuez, Upwind methods for hyperbolic conservation laws with source terms, Comput. \& Fluids, 23 (1994), pp. 1049-1071.

Copyright (C) by SIAM. Unauthorized reproduction of this article is prohibited. 
[6] S. Bian and J.-G. Liu, Dynamic and steady states for multi-dimensional Keller-Segel model with diffusion exponent $m>0$, Commun. Math. Phys., 323 (2013), pp. 1017-1070.

[7] J. Binney and S. Tremaine, Galactic Dynamics, Princeton University Press, Princeton, NJ, 2011.

[8] F. Bouchut, Nonlinear Stability of Finite Volume Methods for Hyperbolic Conservation Laws: And Well-Balanced Schemes for Sources, Springer, Basel, 2004.

[9] V. Calvez, J. A. Carrillo, and F. Hoffmann, Equilibria of homogeneous functionals in the fair-competition regime, Nonlinear Anal., 159 (2017), pp. 85-128.

[10] V. Calvez, J. A. Carrillo, and F. Hoffmann, The geometry of diffusing and self-attracting particles in a one-dimensional fair-competition regime, in Nonlocal and Nonlinear Diffusions and Interactions: New Methods and Directions, Springer, Cham, Switzerland, 2017, pp. $1-71$.

[11] G. CAPDEville, A central WENO scheme for solving hyperbolic conservation laws on nonuniform meshes, J. Comput. Phys., 227 (2008), pp. 2977-3014.

[12] J. A. Carrillo, A. Chertock, and Y. Huang, A finite-volume method for nonlinear nonlocal equations with a gradient flow structure, Commun. Comput. Phys., 17 (2015), pp. 233-258.

[13] J. A. Carrillo, Y.-P. Choi, and S. P. Perez, A review on attractive-repulsive hydrodynamics for consensus in collective behavior, in Active Particles Vol. 1, Springer, Cham, Switzerland, 2017, pp. 259-298.

[14] J. A. Carrillo, Y.-P. Choi, E. Tadmor, and C. TAN, Critical thresholds in $1 d$ Euler equations with non-local forces, Math. Models Methods Appl. Sci., 26 (2016), pp. 185-206.

[15] J. A. Carrillo, E. Feireisl, P. Gwiazda, and A. Świerczewska-Gwiazda, Weak solutions for Euler systems with non-local interactions, J. Lond. Math. Soc. (2), 95 (2017), pp. 705724.

[16] J. A. Carrillo, M. Fornasier, J. Rosado, and G. Toscani, Asymptotic flocking dynamics for the kinetic Cucker-Smale model, SIAM J. Math. Anal., 42 (2010), pp. 218-236.

[17] J. A. Carrillo, M. Fornasier, G. Toscani, and F. Vecil, Particle, kinetic, and hydrodynamic models of swarming, in Mathematical Modeling of Collective Behavior in Socioeconomic and Life Sciences, Springer, Dorcrecht, Netherlands, 2010, pp. 297-336.

[18] J. A. Carrillo, F. Hoffmann, E. Mainini, and B. Volzone, Ground states in the diffusiondominated regime, Calc. Var. Partial Differential Equations, 57 (2018), 127.

[19] J. A. Carrillo, Y. Huang, And S. Martin, Explicit flock solutions for quasi-Morse potentials, European J. Appl. Math., 25 (2014), pp. 553-578.

[20] J. A. Carrillo, S. Kalliadasis, S. P. Perez, and C.-W. Shu, Well-balanced finite-volume schemes for hydrodynamic equations with general free energy, 2020, Multiscale Model. Simul., 18 (2020), pp. 502-541.

[21] J. A. Carrillo, A. Wróblewska-Kamiśska, and E. Zatorska, On long-time asymptotics for viscous hydrodynamic models of collective behavior with damping and nonlocal interactions, Math. Models Methods Appl. Sci., 29 (2018), pp. 31-63.

[22] M. Castro, J. Gallardo, and C. Parés, High order finite volume schemes based on reconstruction of states for solving hyperbolic systems with nonconservative products. Applications to shallow-water systems, Math. Comput., 75 (2006), pp. 1103-1134.

[23] M. J. CAstro, T. M. DE Luna, And C. PARÉS, Well-balanced schemes and path-conservative numerical methods, in Handbook of Numerical Analysis, Vol. 18, Elsevier, Amsterdam, 2017, pp. 131-175.

[24] M. J. Castro, J. A. López-García, And C. Parés, High order exactly well-balanced numerical methods for shallow water systems, J. Comput. Phys., 246 (2013), pp. 242-264, https: //doi.org/10.1016/j.jcp.2013.03.033.

[25] M. J. Castro, A. Pardo Milanés, and C. Parés, Well-balanced numerical schemes based on a generalized hydrostatic reconstruction technique, Math. Models Methods Appl. Sci., 17 (2007), pp. 2055-2113.

[26] M. J. CASTRO AND C. PARÉs, Well-balanced high-order finite volume methods for systems of balance laws, J. Sci. Comput., 82 (2020), 48, https://doi.org/10.1007/s10915-020-01149-5.

[27] M. J. Castro AND M. Semplice, Third-and fourth-order well-balanced schemes for the shallow water equations based on the CWENO reconstruction, Internat. J. Numer. Methods Fluids, 89 (2019), pp. 304-325.

[28] C. Chalons, P. Goatin, and L. M. Villada, High-order numerical schemes for onedimensional nonlocal conservation laws, SIAM J. Sci. Comput., 40 (2018), pp. A288-A305.

[29] P.-H. Chavanis And C. SiRe, Kinetic and hydrodynamic models of chemotactic aggregation, Phys. A, 384 (2007), pp. 199-222.

[30] Y. Cheng, A. Chertock, M. Herty, A. Kurganov, and T. Wu, A new approach for designing moving-water equilibria preserving schemes for the shallow water equations, J. Sci. Comput., 80 (2019), pp. 538-554.

Copyright $@$ by SIAM. Unauthorized reproduction of this article is prohibited. 
[31] A. Chertock, S. Cui, A. Kurganov, S.. N. Özcan, and E. Tadmor, Well-balanced schemes for the Euler equations with gravitation: Conservative formulation using global fluxes, J. Comput. Phys., 358 (2018), pp. 36-52.

[32] Y.-P. CHOI, S.-Y. HA, AND Z. LI, Emergent dynamics of the Cucker-Smale flocking model and its variants, in Active Particles, Vol. 1, Springer, Cham, Switzerland, 2017, pp. 299-331.

[33] V. Churuksaeva And A. Starchenko, Mathematical modeling of a river stream based on a shallow water approach, Procedia Comput. Sci., 66 (2015), pp. 200-209.

[34] S. Cordier AND E. Grenier, Quasineutral limit of an Euler-Poisson system arising from plasma physics, Comm. Partial Differential Equations, 25 (2000), pp. 1099-1113.

[35] L. Cozzolino, V. Pepe, L. Cimorelli, A. D'Aniello, R. Della Morte, and D. Pianese, The solution of the dam-break problem in the porous shallow water equations, Adv. Water Res., 114 (2018), pp. 83-101.

[36] F. Cucker and S. Smale, Emergent behavior in flocks, IEEE Trans. Automat. Control, 52 (2007), pp. 852-862.

[37] F. Cucker And S. Smale, On the mathematics of emergence, Jpn. J. Math., 2 (2007), pp. 197227.

[38] F. Cucker, S. Smale, And D.-X. Zhou, Modeling language evolution, Found. Comput. Math., 4 (2004), pp. 315-343.

[39] F. Filbet, A finite volume scheme for the Patlak-Keller-Segel chemotaxis model, Numer. Math., 104 (2006), pp. 457-488.

[40] F. Filbet And C.-W. Shu, Approximation of hyperbolic models for chemosensitive movement, SIAM J. Sci. Comput., 27 (2005), pp. 850-872.

[41] U. S. Fjordholm, S. Mishra, and E. Tadmor, Arbitrarily high-order accurate entropy stable essentially nonoscillatory schemes for systems of conservation laws, SIAM J. Numer. Anal., 50 (2012), pp. 544-573.

[42] J. M. Gallardo, C. Parés, And M. J. Castro, On a well-balanced high-order finite volume scheme for shallow water equations with topography and dry areas, J. Comput. Phys., 227 (2007), pp. 574-601.

[43] A. Gamba, D. Ambrosi, A. Coniglio, A. De Candia, S. Di Talia, E. Giraudo, G. Serini, L. Preziosi, and F. Bussolino, Percolation, morphogenesis, and Burgers dynamics in blood vessels formation, Phys. Rev. Lett., 90 (2003), 118101.

[44] L. GASCón AND J. CoRberán, Construction of second-order TVD schemes for nonhomogeneous hyperbolic conservation laws, J. Comput. Phys., 172 (2001), pp. 261-297.

[45] I. GIARDINA, Collective behavior in animal groups: Theoretical models and empirical studies, HFSP Journal, 2 (2008), pp. 205-219.

[46] J. Giesselmann, C. Lattanzio, and A. E. Tzavaras, Relative energy for the Korteweg theory and related Hamiltonian flows in gas dynamics, Archive Ration. Mech. Anal., 223 (2017), pp. $1427-1484$

[47] B. D. Goddard, A. Nold, N. Savva, G. A. Pavliotis, and S. Kalliadasis, General dynamical density functional theory for classical fluids, Phys. Rev. Lett., 3 (2012), 120603.

[48] B. D. Goddard, A. Nold, N. Savva, P. Yatsyshin, and S. Kalliadasis, Unification of dynamic density functional theory for colloidal fluids to include inertia and hydrodynamic interactions: derivation and numerical experiments, J. Phys. Condensed Matter, 25 (2013), 035101.

[49] L. Gosse, A well-balanced flux-vector splitting scheme designed for hyperbolic systems of conservation laws with source terms, Comput. Math. Appl., 39 (2000), pp. 135-159.

[50] L. Gosse, Asymptotic-preserving and well-balanced schemes for the 1d Cattaneo model of chemotaxis movement in both hyperbolic and diffusive regimes, J. Math. Anal. Appl., 388 (2012), pp. 964-983.

[51] S. Gottlieb And C.-W. Shu, Total variation diminishing Runge-Kutta schemes, Math. Comput., 67 (1998), pp. 73-85.

[52] J. M. Greenberg And A. Y. LeRoux, A well-balanced scheme for the numerical processing of source terms in hyperbolic equations, SIAM J. Numer. Anal., 33 (1996), pp. 1-16.

[53] Y. Guo And B. Pausader, Global smooth ion dynamics in the Euler-Poisson system, Comm. Math. Phys., 303 (2011), pp. 89-125.

[54] S.-Y. HA AND E. TADMOR, From particle to kinetic and hydrodynamic descriptions of flocking, Kinet. Relat. Models, 1 (2008), pp. 415-435.

[55] M. Hadžić And J. J. JANG, A class of global solutions to the Euler-Poisson system, Comm. Math. Phys., 370 (2019), pp. 475-505.

[56] H. Hildenbrandt, C. Carere, and C. K. Hemelrijk, Self-organized aerial displays of thousands of starlings: A model, Behav. Ecol., 21 (2010), pp. 1349-1359.

Copyright (C) by SIAM. Unauthorized reproduction of this article is prohibited. 
[57] Y. Katz, K. Tunstrøm, C. C. Ionnnou, C. Huepe, and I. D. Couzin, Inferring the structure and dynamics of interactions in schooling fish, Proc. Natl. Acad. Sci. USA, 108 (2011), pp. $18720-18725$.

[58] E. F. Keller and L. A. Segel, Initiation of slime mold aggregation viewed as an instability, J. Theoret. Biol., 26 (1970), pp. 399-415.

[59] C. Klingenberg, G. Puppo, And M. Semplice, Arbitrary order finite volume well-balanced schemes for the Euler equations with gravity, SIAM J. Sci. Comput., 41 (2019), pp. A695A721.

[60] C. Lattanzio and A. E. Tzavaras, From gas dynamics with large friction to gradient flows describing diffusion theories, Comm. Partial Differential Equations, 42 (2017), pp. 261-290.

[61] D. Levy, G. Puppo, and G. Russo, Central WENO schemes for hyperbolic systems of conservation laws, ESAIM Math. Model. Numer. Anal., 33 (1999), pp. 547-571.

[62] D. Levy, G. Puppo, And G. Russo, Compact central WENO schemes for multidimensional conservation laws, SIAM J. Sci. Comput., 22 (2000), pp. 656-672.

[63] F. Marche, P. Bonneton, P. Fabrie, and N. Seguin, Evaluation of well-balanced borecapturing schemes for $2 d$ wetting and drying processes, Internat. J. Numer. Methods Fluids, 53 (2007), pp. 867-894.

[64] P. Minakowski, P. B. Mucha, J. Peszek, and E. Zatorska, Singular Cucker-Smale dynamics, in Active Particles, Volume 2, Springer, 2019, pp. 201-243.

[65] S. Motsch AND E. TADMOR, A new model for self-organized dynamics and its flocking behavior, J. Stat. Phys., 144 (2011), 923.

[66] R. Natalini And M. Ribot, Asymptotic high order mass-preserving schemes for a hyperbolic model of chemotaxis, SIAM J. Numer. Anal., 50 (2012), pp. 883-905.

[67] R. Natalini, M. Ribot, and M. Twarogowska, A Well-Balanced Numerical Scheme for a One Dimensional Quasilinear Hyperbolic Model of Chemotaxis, Commun. Math. Sci., 12 (2014), pp. 13-39.

[68] S. Noelle, N. Pankratz, G. Puppo, and J. R. Natvig, Well-balanced finite volume schemes of arbitrary order of accuracy for shallow water flows, J. Comput. Phys., 213 (2006), pp. 474-499.

[69] S. Noelle, Y. XING, AND C.-W. Shu, High-order well-balanced finite volume WENO schemes for shallow water equation with moving water, J. Comput. Phys., 226 (2007), pp. 29-58.

[70] L. Perea, G. Gómez, and P. Elosegui, Extension of the Cucker-Smale control law to space flight formations, J. Guid. Control Dyn., 32 (2009), pp. 527-537.

[71] B. Perthame and C. Simeoni, A kinetic scheme for the Saint-Venant system with a source term, Calcolo, 38 (2001), pp. 201-231.

[72] G. Serini, D. Ambrosi, E. Giraudo, A. Gamba, L. Preziosi, and F. Bussolino, Modeling the early stages of vascular network assembly, EMBO J., 22 (2003), pp. 1771-1779.

[73] M. B. Short, M. R. D'orsogna, V. B. Pasour, G. E. Tita, P. J. Brantingham, A. L. Bertozzi, and L. B. Chayes, A statistical model of criminal behavior, Math. Models Methods Appl. Sci., 18 (2008), pp. 1249-1267.

[74] C.-W. SHu, Essentially Non-oscillatory and Weighted Essentially Non-oscillatory Schemes for Hyperbolic Conservation Laws, Technical report, NASA/CR-97-206253, NASA Langley Research Center, Hampton, VA, 1997.

[75] C.-W. Shu AND S. Osher, Efficient implementation of essentially non-oscillatory shockcapturing schemes, J, Comput. Phys., 77 (1988), pp. 439-471, https://doi.org/10.1016/ 0021-9991(88)90177-5.

[76] C.-W. Shu AND S. Osher, Efficient implementation of essentially non-oscillatory shockcapturing schemes, II, J. Comput. Phys., 83 (1989), pp. 32-78, https://doi.org/10.1016/ 0021-9991(89)90222-2.

[77] E. TADMOR, Entropy stable schemes, in Handbook of Numerical Analysis, Vol. 17, Elsevier, Amsterdam, 2016, pp. 467-493.

[78] A. Thomann, M. Zenk, And C. Klingenberg, A second-order positivity-preserving wellbalanced finite volume scheme for Euler equations with gravity for arbitrary hydrostatic equilibria, Internat. J. Numer. Methods Fluids, 89 (2019), pp. 465-482.

[79] Y. XING AND C.-W. SHu, High order well-balanced finite volume WENO schemes and discontinuous Galerkin methods for a class of hyperbolic systems with source terms, J. Comput. Phys., 214 (2006), pp. 567-598.

[80] Y. XING AND C.-W. SHU, High order well-balanced WENO scheme for the gas dynamics equations under gravitational fields, J. Sci. Comput., 54 (2013), pp. 645-662.

[81] Y. XING AND C.-W. Shu, A survey of high order methods for the shallow water equations, J. Math. Study, 47 (2014), pp. 221-249.

[82] X. Zhang And C.-W. ShU, On maximum-principle-satisfying high order schemes for scalar conservation laws, J. Comput. Phys., 229 (2010), pp. 3091-3120.

Copyright $@$ by SIAM. Unauthorized reproduction of this article is prohibited. 